\title{
Effects of Fueling Profiles on Plasma Transport
}
A. T. Mense
W. A. Houlberg
S. E. Attenberger
S. L. Milora
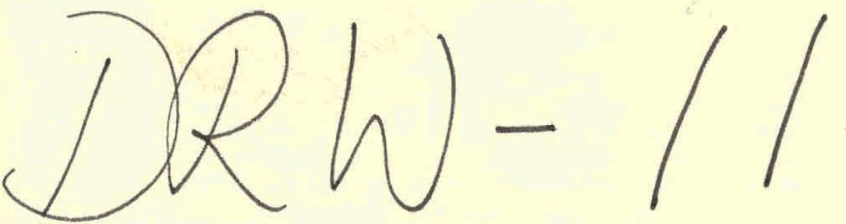


\section{DISCLAIMER}

This report was prepared as an account of work sponsored by an agency of the United States Government. Neither the United States Government nor any agency Thereof, nor any of their employees, makes any warranty, express or implied, or assumes any legal liability or responsibility for the accuracy, completeness, or usefulness of any information, apparatus, product, or process disclosed, or represents that its use would not infringe privately owned rights. Reference herein to any specific commercial product, process, or service by trade name, trademark, manufacturer, or otherwise does not necessarily constitute or imply its endorsement, recommendation, or favoring by the United States Government or any agency thereof. The views and opinions of authors expressed herein do not necessarily state or reflect those of the United States Government or any agency thereof. 


\section{DISCLAIMER}

Portions of this document may be illegible in electronic image products. Images are produced from the best available original document. 
Printed in the United States of America. Available from National Technical Information Service

U.S. Department of Commerce

5285 Port Royal Road, Springfield, Virginia 22161

Price: Printed Copy $\$ 6.00$; Microfiche $\$ 3.00$

\subsection{5}

This report was prepared as an account of work sponsored by an agency of the United States Government. Neither the United States Government nor any agency thereof, nor any of their employees, contractors, subcontractors, or their employees, makes any warranty, express or implied, nor assumes any legal liability or responsibility for any third party's use or the results of such use of any information, apparatus, product or process disclosed in this report, nor represents that its use by such third party would not infringe privately owned rights. 
Contract No. W-7405-eng-26

\author{
FUSION ENERGY DIVISION
}

EFFECTS OF FUELING PROFILES ON PLASMA TRANSPORT

A. T. Mense, W. A. Houlberg, S. E. Attenberger, and S. L. Milora

Date Published: April 1978

NOTICE This document contains information of a preliminary nature. It is subject to revision or correction and therefore does not represent a final report.

Prepared by the OAK RIDGE NATIONAL LABORATORY Oak Ridge, Tennessee 37830 operated by UNION CARBIDE CORPORATION

for the DEPARTMENT OF ENERGY

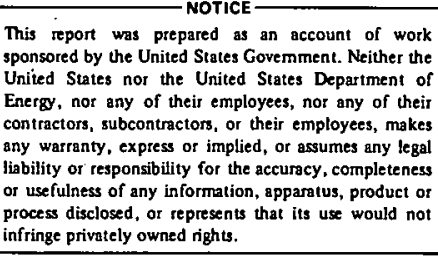

This report was prepared as an account of work sponsoped by the United States Govemment. Neither the Energy, not any of their employees, nor any of their contractors, subcontractors, or their employees, makes any wastanty, express or implied, or assumes any legal or usefulness of any information, appanatus, product of process disclosed, or represents
infringe privately owned rights. 


\section{THIS PAGE}

\section{WAS INTENTIONALLY \\ LEFT BLANK}


ABSTRACT ........................ . . v

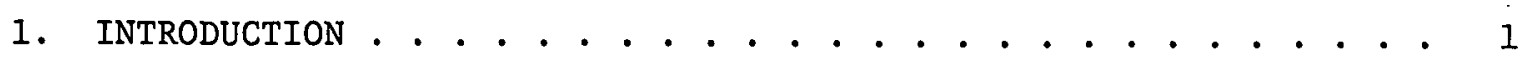

2. THE MULTIFLUID PARTICLE AND ENERGY TRANSPORT MODEL . . . . . . 3

3. THE PELLET MODEL . . . . . . . . . . . . . . . . . 13

4. FUELING PROFILE EFFECTS ON IGNITED PLASMA EQUILIBRIA . . . . . 18

5. EFFECTS OF CONTINUOUS FUELING PROFILES ON PLASMA TRANSPORT

AND IGNITION . . . . . . . . . . . . . . . . . 28

6. PELLET INJECTION SIMULATION . . . . . . . . . . . . . 41

7. SUMMARY . . . . . . . . . . . . . . . 51

APPENDIX A. BEHAVIOR OF THE PARTICLE BALANCE IN TOKAMAKS UNDER THE CONDITIONS OF EDGE FUELING . . . . . . . . . . . . . . 54

APPENDIX B. DATA FROM EQUILIBRIUM PROFILE STUDY . . . . . . . . 57 ACKNOWLEDGMENTS . . . . . . . . . . . . . . . . . . 63

REFERENCES . . . . . . . . . . . . . . . . . . 64 
THIS PAGE

WAS INTENTIONALLY

LEFT BLANK 
ABSTRACT

A one-dimensional (1-D), multifluid transport model is used to investigate the effects of particle fueling profiles on plasma transport in an ignition-sized tokamak (TNS). Normal diffusive properties of plasmas will likely maintain the density at the center of the discharge even if no active fueling is provided there. This significantly relaxes the requirements for fuel penetration. Not only is lower fuel penetration easier to achieve, but it may have the advantage of reducing or eliminating density gradient-driven trapped particle microinstabilities. Simulation of discrete pellet fueling indicates that relatively low velocity $\left(\sim 10^{3} \mathrm{~m} / \mathrm{sec}\right)$ pellets may be sufficient to fuel a TNS-sized device $(\sim 1.25-\mathrm{m}$ minor radius), to produce a relatively broad, cool edge region of plasma which should reduce the potential for sputtering, and also to reduce the likelihood of trapped particle mode dominated transport. Low penetrating pellets containing up to $10-20 \%$ of the total plasma ions can produce fluctuations in density and temperature at the plasma edge, but the pressure profile and fusion alpha production remain almost constant. 


\section{INTRODUCTION}

This report summarizes the results of initial investigations of the effects of particle fueling profiles on the particle and energy transport characteristics of an ignition-sized tokamak operating with a poloidal divertor. A set of TNS parameters, given in Table 1, is used as representative for such a device. The study was conducted with a 1-D. multifluid transport code.(WHIST), discussed in Sect. 2. Particular attention is given in this study to the way in which fueling profiles affect the particle and energy loss rates due to low frequency density gradient-driven microinstabilities (trapped particle modes). Expressions from WASH-1295 ${ }^{1}$ are used for the trapped particle modes on the basis of familiarity with the transport coefficients in the general fusion community. These modes have not been identified as responsible for transport in current tokamak plasmas, but nevertheless they exhibit unfavorable scaling with temperature and have been used to provide an approximate upper estimate on heating-to-ignition power requirements.: Neoclassical expressions from the work of Hinton and Moore ${ }^{2}$ are used as a lower bound on the particle and energy transport.' Further discussion of the transport models is given in Sect. 2 .

One of the most interesting aspects of this study centers on initial investigations of particle fueling effects with discrete pellets: A pellet ablation code developed by Milora and Foster ${ }^{3}$ has been incorporated in the $1-D$, time-dependent, fluid transport code used for this study. This pe11et model has been benchmarked against ORMAK experimental results. ${ }^{3}$ A discussion of the pellet ablation physics is contained in sect. 3 . Discrete pellets of a given size and velocity are injected into the plasma and their deposition profiles are determined self-consistently with the plasma density and temperature profiles. The pellet deposition profile in turn affects the density and temperature profiles through the particle and energy halances.

The results of the study, which involves three phases, are contained in Sects. 4-6. The first phase of the study (Sect. 4) is a brief investigation of the effect of particle fueling profiles on thermally stable ignited equilibria during steady-state burn. An assumed analytic source profile linearly decreasing in intensity from the plasma edge is used. 
Table 1. TNS parameters

$\begin{array}{ll}\text { Major radius } & \mathrm{R}=500 \mathrm{~cm} \\ \text { Minor radius in midplane } & \mathrm{a}=125 \mathrm{~cm} \\ \text { Plasma elongation } & \mathrm{b} / \mathrm{a}=1.6 \\ \text { Minor radius of equivalent circle } & \mathrm{a}_{\text {eff }}=160 \mathrm{~cm} \\ \text { Toroidal magnetic field } & \mathrm{B}_{\mathrm{T}}=4.3 \mathrm{~T} \\ \text { Toroidal current } & \mathrm{I}=4 \times 10^{6} \mathrm{~A}\end{array}$

Donoity and tempcrature boundary cundllluns:

$$
\begin{aligned}
& \Delta_{n_{\alpha, i}} \equiv\left(-\frac{1}{\mathrm{n}} \frac{\partial \mathrm{n}}{\partial \mathrm{r}}\right)^{-1}=10 \mathrm{~cm} \\
& \therefore \Delta_{\mathrm{T}_{\mathrm{e}}} \equiv\left(-\frac{1}{\mathrm{~T}_{\mathrm{e}}} \frac{\partial \mathrm{T}}{\partial \mathrm{r}}\right)^{-1}=5 \mathrm{~cm} \\
& v^{-1} \equiv\left(-\frac{1}{\mathrm{~T}_{i}} \frac{\partial \mathrm{T}_{i}}{\partial \mathrm{r}}\right)^{-1}=10 \mathrm{~cm} \\
& \Delta_{\mathrm{T}_{i}}
\end{aligned}
$$


The dependence of the equilibrium plasma parameters on source penetration depth and plasma density is discussed.

Greater emphasis is given to the analysis of the way in which particle fueling profiles affect ignition requirements during startup (Sects. 5 and 6) than is given to the way fueling profiles affect equilibrium burn behavior because of the lesser degree of physics extrapolation required for the study. In Sect. 5 analytic source profiles are used to determine the effects of continuous fueling profiles on minimum power requirements for ignition.

The main body of the work, which looks at the effects of discrete pellet fueling on ignition requirements, is discussed in Sect. 6 . Previous estimates of required pellet velocities for large tokamaks included the assumption that the pellets must penetrate to the center of the discharge. Analysis of expeximental results and available transport models indicates that this assumption is conservative. Reducing the pellet penetration depth by a factor of two can decrease the required pellet velocities by nearly an order of magnitude. ${ }^{3}$

Section 7 outlines the conclusions which may be drawn from this work along with a restatement of the caveats of the models used. Future directions for both theoretical and experimental work are also discussed.

\section{THE MULTIFLUID PARTICLE AND ENERGY TRANSPORT MODEL}

A one-dimensional fluid particle and energy model is used for the thermal components of the plasma. Two ionic species are modeled: a 50-50 $D-T$ component and a thermal alpha component. The electron density is treated as a dependent variable by requiring charge neutrality. The density and energy balance equations and brief explanations of each sink, source, and transport term are presented here. The density equations are:

$$
\frac{\partial n_{i}}{\partial t}=-\frac{1}{r} \frac{\partial}{\partial r}\left(r \Gamma_{i}\right)+s_{p}+s_{b}-\frac{n_{i}^{2}\langle\sigma v\rangle_{D T}}{2}-2 s_{b f}
$$




$$
\begin{aligned}
& \frac{\partial n_{\alpha}}{\partial t}=-\frac{1}{r} \frac{\partial}{\partial r}\left(r \Gamma_{\alpha}\right)+\frac{n_{i}^{2}\langle\sigma v\rangle}{2}+s_{b f} \\
& n_{e}=n_{i}+2 n_{\alpha} \\
& \Gamma_{e}=\Gamma_{i}+2 \Gamma_{\alpha},
\end{aligned}
$$

where

$$
\begin{aligned}
n_{i, \alpha, e} & =\text { hydrogenic ion, alpha, and electron densities } \\
\Gamma_{i, n, e} & =\text { hydrogenic ion, alpha, and eleclrun particle fluxes } \\
\mathrm{s}_{\mathrm{p}} & =\text { pellet source of hydrogenic ions } \\
\mathrm{S}_{\mathrm{b}} & =\text { beam source of hydrogenlc ions } \\
\mathrm{S}_{\mathrm{bf}} & =\text { rate of beam-plasma fusions. }
\end{aligned}
$$

Fluid energy balance equations are used for the thermal components of hydrogenic ions, alphas, and electrons. The thermal component of the alphas is assumed to thermalize rapidly with the hydrogenic ions because of the nearly equal masses. Inder this aseumption the thermal iuns dud alphas should have nearly the same local temperature. The thermal ion and alpha energy balance equations are added to remove the fast rethermalization term and obtain a "lumped ion" energy balance in terms of the ionic temperature, $T_{i}$. The two fluid energy balance equations are then given by:

$$
\begin{aligned}
\frac{\partial}{\partial t}\left(\frac{3}{2} n_{e} T_{e}\right) & =\left(\frac{n_{i}^{2} \sigma v>D T}{4}+S_{b f}\right) E_{\alpha} U_{\alpha e}-\frac{1}{r} \frac{\partial}{\partial r}\left[r\left(Q_{e}+\frac{3}{2} T_{e} \Gamma_{e}\right)\right] \\
& -Q_{e i}-Q_{e \alpha}-P_{r a d}+P_{o h m}+P_{i n j}^{e}
\end{aligned}
$$




$$
\begin{aligned}
\frac{\partial}{\partial t}\left[\frac{3}{2}\left(n_{i}+n_{\alpha}\right) T_{i}\right] & =\left(\frac{n_{1}^{2}\left\langle\sigma v D_{D T}\right.}{4}+S_{b f}\right) E_{\alpha}\left(1-U_{\alpha e}\right)-\frac{1}{r} \frac{\partial}{\partial r}\left[r\left(Q_{i}+\frac{3}{2} T_{i} \Gamma_{i}\right)\right] \\
& -\frac{1}{r} \frac{\partial}{\partial r}\left[r\left(Q_{\alpha}+\frac{3}{2} T_{i} \Gamma_{\alpha}\right)\right]+Q_{e i}+Q_{e \alpha}+P_{i n j}^{i}+P_{i n j}^{\alpha},
\end{aligned}
$$

where

$$
\begin{aligned}
& E_{\alpha}=3.52 \mathrm{MeV}, \text { fusion alpha energy } \\
& U_{\alpha e}=\text { fraction of alpha energy given to electrons } \\
& Q_{i ; \alpha, e}=\text { hydrogenic ion, alpha, and electron conduction energy } \\
& \text { fluxes } \\
& Q_{e i}, Q_{e \alpha}=\text { electron-ion and electron-thermal alpha rethermalization } \\
& P_{\text {rad }}=\text { radiation losses } \\
& P_{o h m}=\text { ohmic energy source } \\
& P_{i, \alpha, e}^{i}=\text { energy source to hydrogenic ions, thermal alphas, and } \\
& \text { electrons from high energy beams. }
\end{aligned}
$$

The rethermalization energy flows between electrons and the thermal ions are calculated from clasical Coulomb collisions. ${ }^{4}$

$Q_{e j}=3 \frac{n_{c}}{\tau_{e j}} \frac{m_{c}}{m_{j}}\left(T_{e}-T_{i}\right) \cdot j=i, \alpha$

$$
\tau_{e j}=3.47 \times 10^{8} \frac{T_{e}^{3 / 2}}{Z_{j}^{z} n_{j} \ln \Lambda} \operatorname{msec} \quad j=i, \alpha .
$$

For the cases considered in this study, the ohmic heating source is small compared to the neutral beam injection power and the heating from fusion alphas. Not included in this analysis are very early phases of the discharge which encompass: (1) initial gas breakdown; (2) heating through 
the ionization states of hydrogen and possibly oxygen, and other 1ow-Z impurities; and (3) penetration of the toroidal plasma current. The above considerations alone require a much more detailed startup scenario for the relatively large plasmas considered here. The transport calculations are begun after these early stages with temperatures of $\sim 200 \mathrm{eV}$ and a parabolic toroidal current profile. Proper treatment of the evolution of the current profile should include current diffusion and coupling to MHD equations. For simplicity, the current profile was fixed in time with the local resistivity and ohmic heating being calculated using neoclassical corrections to spitzer resistivity. 5

$P_{\text {ohm }}=n J^{2}$

$n(r)=n_{s p}\left[1-1.95(r / R)^{1 / 2}+0.95(r / R)\right]^{-1}$

$\eta_{s p}=1.02 \times 10^{-2} \frac{z_{\text {eff }}^{\ell n \Lambda}}{T_{e}^{3 / 2}}\left(0.291: \frac{0.45 Z}{1.007+z_{\text {eff }}}\right) s l-c m$

( $\mathrm{T}_{\mathrm{e}}$ measured in $\left.\mathrm{eV}\right)$.

It is assumed that a poloidal divertor, which is discussed further in SecL. 4, w111 effectively prevent impurities from entering the plasma and will act as a pump for the particles diffusing from the system. 6 The radiation losses from the clean plasma consist of normal bremsstrahlung plus an approximate form for synchrotron radiation. ${ }^{7}$

$$
P_{B}=9.48 \times 10^{-17} \mathrm{n}^{2} \mathrm{Z} \mathrm{eff}^{\mathrm{T}^{1 / 2}} \mathrm{eV} / \mathrm{cm}^{3} / \mathrm{msec}
$$


$\mathrm{P}_{\mathrm{SYN}}=6.09 \times 10^{-13}\left[1+0.18\left(1-\frac{\mathrm{R}}{5 \mathrm{a}}\right)\right]^{3} \mathrm{~B}_{\mathrm{T}}^{5 / 2} \mathrm{~T}_{\mathrm{e}}^{2 \cdot 1} \frac{\mathrm{n}^{1 / 2}}{\mathrm{e}} \cdot \mathrm{eV} / \mathrm{cm}^{3} / \mathrm{msec}$

$P_{\text {rad }}=P_{B}+P_{S Y N}$,

where the units are $\mathrm{T}_{\mathrm{e}}(\mathrm{eV}), \mathrm{n}_{\mathrm{e}}\left(\mathrm{cm}^{-3}\right), \mathrm{B}_{\mathrm{T}}(\mathrm{G}), \mathrm{a}(\mathrm{cm})$, and $\mathrm{R}(\mathrm{cm})$.

A "pencil beam" model is used for the neutral beam in these initial studies with the beam path lying in the plasma midplane. More detailed finite beam calculations would likely provide modest changes in the beam deposition profile. Beam attenuation is primarily due to ion impact ionization, but charge exchange and electron impact ionization are also included. The beam penetration is corrected for the noncircular plasma cross section by mapping the density and temperature profiles from the equivalent circular cross section onto the smaller grid in the plasma midplane. The penetration is not corrected for outward flux surface shifts which are due to finite $\beta$ effects, 8 since the $\beta$ is still relatively low when the beams are turned off at ignition. The thermalization of the fast beam ions occurs on a short time scale ( $\sim 200 \mathrm{msec}$ ) compared to the time variation of the plasma parameters ( $\imath_{1}$ sec). The energy deposition is assumed to be local (on the flux surface where the neutral is ionized) with the partition of energy between the electron and "1umped ion" fluids determined from classical Coulomb collisions. The fast beam ions are very collisionless and would not be expected to diffuse very far in radius with a collisional diffusion model such as neoclassical theory. Anomalous diffusion of the fast ions would broaden the heating profile relative to the deposition profile with possible loss of those ions trapped near the plasma edge. So far no microinstabilities driven by the fast beam component have been observed in neutral injection experiments. ${ }^{9}$ Finite orbit effects of the fast beam ions are also not included in the energy deposition profile and should provide only moderate corrections in the TNS-size plasma considered here. The model for the fast ion thermalization is given by: 10 


$$
\begin{aligned}
& \frac{d E_{f}^{3 / 2}}{d t}=-\frac{1}{\tau}\left(E_{c}^{3 / 2}+E_{f}^{3 / 2}\right) \\
& E_{c}=14.8 T_{e} A_{f}\left(\sum_{j=i o n s} \frac{z_{i}^{2} n_{j}}{A_{j} n_{e}}\right)^{2 / 3} \\
& \tau=2.1 \times 10^{11} \frac{A_{f}}{Z_{f}^{2}} \frac{T_{e}^{3 / 2}}{n_{e}^{\ell n} \Lambda} \text { msec },
\end{aligned}
$$

where $\mathrm{E}_{f}, \mathrm{~A}_{f}$, and $\mathrm{Z}_{f}$ are the mean energy, mass number, and charge of the fast test particle. The units are eV, $\mathrm{cm}$, and $\mathrm{msec}$ for all. parameters. Diffusion in velocity space about the mean energy has been neglected. Equation (2.13) has the solution

$$
E_{f}^{3 / 2}(t)=E_{f o}^{3 / 2} e^{-t / \tau}-E_{c}^{3 / 2}\left(1-e^{-t / \tau}\right)
$$

$E_{f}$ represents the mean energy of the fast particle as a function of time. The fraction of energy given to the electrons is a function of $E_{c}$ and the initial and final test particle energies $E_{\text {fo }}$ and $E_{f}$, respectively:

$$
U_{b e}=1-\frac{1}{E_{[u}-E_{f}} \int_{E_{f}}^{E_{f o}} \frac{E_{c}^{3 / 2}}{E_{f^{\prime 3 / 2}}^{\prime 3}+E_{C}^{3 / 2}} d E_{f}^{\prime} .
$$

The probability of the fast beam particles producing fusions while slowing down is calculated approximately by assuming that the background ions are cold and the relative velocity between beam and plasma ions is given by the fast ion velocity. ${ }^{11}$ For a deuterium beam the rate of beamplasma fusions is given by: 
$S_{b f}=n_{T} \int v \sigma_{D T}(v) f_{D}(v) d^{3} v$

where $f_{D}$ represents the distribution function of fast deuterium ions and $\mathrm{n}_{\mathrm{T}}$ represents the density of thermal tritium.

Cordey, Core, and Sheffield ${ }^{12}$ evaluated the effects of neglecting velocity space diffusion and finite thermal ion temperature on the beamplasma fusion rate and found both effects to be fairly small. For a deuterium beam at $150 \mathrm{keV}$ with electron temperatures in the range $5-10 \mathrm{keV}$, an increase of $5-10 \%$ in the fusion probability (due to velocity space diffusion) was found. Finite ion temperature corrections give $\check{\imath}_{2 \%}$ increase in the fusion rate for the same parameters.

The fusion alphas are treated in much the same manner as are the fast beam ions. The model for thermalization is given by the classical expressions, Eqs. $(2.13,2.14,2.15)$. They are assumed to thermalize on the surface where they are born, which is consistent with a classical model for thermalization and diffusion. Finite orbit effects for the fast alphas ${ }^{3}$ should be more important than for the fast beam ions because of the alphas' higher energy. The energy deposition profile may be greatly broadened from the production profile. $\left(n^{2}\langle\sigma v\rangle\right)$ but is neglected in the study. Finite thermalization time for the fast alphas may introduce a significant delay time in the heating rate, especially at low plasma density. The finite thermalization time has been modeled in the onedimensional space-time treatment using Eqs. $(2.16,2.17) .14$ The source of fast alphas is stored as a function of both space and time. At subsequent time intervals the energy of the fast alphas is approximated using Eq. (2.16) with the local expressions for $E_{c}$ and $\tau$ given by Eqs. (2.14, 2.15).

The partition of the energy given to electrons and ions is evaluated from Eq. (2.17) at each time interval and at each radius. Microinstabilities driven by the fast alpha distribution may signiticantly reduce the thermalization time and enhance the radial transport of the fast alphas, 15 but this theory was not included in the analysis here.

Several options for the particle source model are used, including fixed or time-dependent radial profiles and continuous or discrete fueling 
rates. The amplitude of the source strength is adjusted in time to maintain approximately constant density. 14 For the continuous fueling models, the source amplitude is adjusted via feedback on a time scale $\tau_{s}$ to maintain the desired average density $\bar{n}_{0}$. The algorithm for determining the particle source strength for the $k$ to $k+1$ timestep is given by:

$\left\{\bar{s}_{p}^{(k+1)}-\bar{s}_{p}^{(k)},\left[\frac{\bar{n}_{0}-2 \bar{n}^{(k)}+\bar{n}^{(k-1)}}{\tau_{s}}\right]\right\}$,

where $k$ is the Index dennting the time interval, and the bar indicates volume-averaged quantities. With the discrete fueling model, a pellet is introduced into the plasma whenever the average density falls below $\overline{\mathrm{n}}_{\mathrm{O}}$.

The particle and energy transport consists of three basic models which are additive: a neoclassical model, a pseudoclassical model, and a trapped particle microinstability model. The neoclassical model is that of Hinton and Moore ${ }^{2}$ for a multispecies plasma in the banana-plateau regime. A11 terms are included except the Ware pinch terms, and this model represents an irreducible lower limit on the particl.e and energy Lidulspurl. A simpliselc multiregime model ${ }^{1}$ is used for pseudoclassical, trapped electron, and trapped ion modes. This model is "diagonal," which assumes that the ion and thermal alpha particle diffusions are dnwn their respective density gradients and the electron and in thermal conductiono are down their respective temperature gradients. The limitations of a diagonal model for particle transport are discussed in Appendix A.

The pseudoclassical model includes anomalous diffusion and electron conduction coefficients given by:

$x_{e}^{P C}=C_{o} \nu_{e i} \rho_{e \theta}^{2}$ 


$$
\begin{aligned}
& D^{P C}=C_{1} \nu_{e i} \rho_{e}^{2} \theta \\
& \nu_{e i}=\text { Coulomb collision frequency for } 90^{\circ} \text { scattering } \\
& \rho_{e \theta}=\text { electron gyroradius in the poloidal field } \\
& C_{0}=10 \\
& C_{1}=C_{0} / 3 .
\end{aligned}
$$

Two collisionality regimes are used for the dissipative trapped electron mode. In the higher collisionality regime the coefficients are:

$$
\begin{aligned}
& x_{e}^{T E 1}=3 \varepsilon^{3 / 2} r^{2} \frac{\omega_{*} \omega_{*}^{T}}{v_{e i}} \\
& x_{i}^{T E 1}=D^{T E 1}=\varepsilon \chi_{e}^{T E 1},
\end{aligned}
$$

where

$$
\begin{aligned}
\varepsilon & =\mathrm{r} / \mathrm{R} \quad=\text { local inverse aspect ratio } \\
\omega_{*} & =-\frac{\mathrm{kT}}{\mathrm{eBr}} \frac{\mathrm{I}}{\mathrm{n}} \frac{\mathrm{dn}}{\mathrm{dr}}=\text { electron diamagnetic drift frequency } \\
\omega_{*}^{\mathrm{T}} & =-\frac{1}{\mathrm{eBr}} \frac{\partial \mathrm{kT}}{\partial \mathrm{r}}=\text { electron temperature gradient drift frequency. }
\end{aligned}
$$

For the lower collisionality regime the coefficients are:

$$
x_{e}^{\mathrm{TE} 2}=0.06\left(\frac{m_{i}}{m_{e}}\right) \varepsilon^{1 / 2} \eta_{e} \frac{B_{\theta}}{\theta B} \nu_{e i} \rho_{e}^{2}
$$


$\chi_{i}^{\mathrm{TE} 2} \equiv \mathrm{D}^{\mathrm{TE} 2} \equiv \varepsilon \chi_{e}^{\mathrm{TE} 2}$

where

$$
\begin{aligned}
& \eta_{e} \equiv \frac{d \ln \left(T_{e}\right)}{d \ln (n)} \\
& \theta \equiv \frac{r_{n}}{L_{s}}=\text { relative shear strength } \\
& r_{n} \equiv\left(-\frac{1}{n} \frac{\partial n}{\partial r}\right)^{-1}=\text { density gradient scale length } \\
& L_{s} \equiv\left(\frac{\varepsilon}{q^{2}} \frac{\partial q}{\partial r}\right)^{-i}=\text { shear length. }
\end{aligned}
$$

Finally, for the dissipative trapped ion mode:

$D=x_{e}-x_{i}=\frac{\varepsilon^{5 / 2} r^{2} w_{*}^{2}}{\nu_{e i}\left(1+T_{e} / T_{i}\right)^{2}}$

Certainly there is a much better theoretical understanding of the conditions fus Llie unstet of the dissipative trapped particle modes today than there was when the above expressions were first postulated. However, much more work still needs to be done to better evaluate the nonlinear development of these instabilities and the resulting transport. A more up-to-datc summary is provided in Ref. 16.

The one-dimensional fluid equations are solved using conservative spatial differencing (on a nonuniform spatial mesh) for the particle and energy fluxes and a variable implicit time treatment. 17 The plasma is treated as having a circular cross section with the equivalent crosssectional area being given by the noncircular area. That is, the TNS plasma shown in Table 1 is being modeled as a circular plasma with an effective radius of $125 \sqrt{1.6} \simeq 160 \mathrm{~cm}$. The transport coefficients are corrected for noncircular effects through modification of the fraction of 
trapped particles. Extrapolation boundary conditions (Table 1 ). consistent with anticipated divertor operation are used. on the densities and temperatures, at the plasma edge. 6 . The values for: the gradients used in these extrapolated boundary conditions were based on estimates from previous work. 6

The reason for not using the full set of divertor physics equations (except in Sect. 4) in most of our study is due to the requirement imposed by the divertor model terms on the maximum allowable. timestep which the code can take in order to prevent numerical instabilities. This is discussed in detail in Refs. 6 and 17. The gradient conditions in Table 1 would characterize a good unload divertor with reasonable shielding:efficiency for cold neutrals but should not appreciably ablate a pellet passing through the scrape-off zone. Thus, the boundary conditions in effect are a rough approximation to more detailed analysis of the divertor characteristics.

\section{THE PELLET MODEL}

\subsection{THE PHYSICAL MODEL}

The model employed to describe the evaporation of solid hydrogen pellets injected into the tokamak plasma is based on the recent theory of Parks, Turnbull, and Foster ${ }^{18}$ with the modifications proposed by Milora and Foster. ${ }^{3}$ Complete details of this model appear in the references cited; only a brief description is repeated here. The model has as its basis the postu1ate. that a continuum of molecular hydrogen is released at the pellet surface as a response to the heat deposited by incident plasma electrons. The resultant dynamic cloud of hydrogen that surrounds the pellet surface partially shields the pellet by reducing the energy of incident plasma electrons before they impact the surface. In this respect, the physical model is somewhat similar to that recently proposed by Vaslow 19 but differs from earlier treatments by Rose. ${ }^{20}$ Gralnick, 21 and Chang, 22 all of whom assumed that ion pairs are the only species emitted at the pellet surface (or at the ionization surface which is located very close to the peliet surface). 
Preliminary validation of the neutral molecule ablation model has been demonstrated experimentally in recent pellet injection studies conducted on ORMAK. 23 The model accurately reproduces the observed pellet ablation rates for pellets. of differing sizes injected into the warm edge plasma. Verification of the model in the hot, dense plasma of interest here is of course lacking, but it should be noted that the calculated lifetime in such instances is generally in agreement with previously published results. The advantage of the neutral ablation model is that it is not restricted solely to very dense, hot plasmas.

As described in Ref. 3, the pellet ablation rate is given in terms of the local values of electron energy $E_{\theta}(e V)$ and density $n_{0}\left(\mathrm{~cm}^{-3}\right)$ by two coupled ordinary differential equations... The first of these, which incorporates the concept of the shield, expresses the amount of material which the pellet must supply in order to slow electrons down to an arbitrary energy $E_{e_{0}}$ before they strike the pellet surface. In terms of the speed with which the pellet surface recedes into the pellet interior, this shielding requirement reduces to

$\frac{d r_{0}}{d t}=-\frac{7.43 \times 10^{-17}}{r_{0}^{2 / 3}} \frac{n_{e}^{1 / 3}}{M^{1 / 3}} E_{e}^{1 / 6}\left[L\left(\frac{E_{e}}{2}\right)\right]^{1 / 3} \cdot \int_{E_{e}}^{E_{0}} \frac{d E_{e}}{L\left(E_{e}\right)}$.

where $r_{0}$ is the instantaneous pellet radius in $\mathrm{cm}, M$ is the molecular weight of the fuel in $a m u, L\left(E_{e}\right)$ is an energy dependent function representing the cumulative loss of energy of electrons in molecular hydrogen by inelastic collisions, ${ }^{24}$ and $E_{e_{0}}$ is the resultant energy with which primary electrons impact the pellet surface. The function $L\left(E_{e}\right)$ is approximated by the expression

$L\left(E_{e}\right)=\left(2.35 \times 10^{14}+4 \times 10^{11} E_{e}+\frac{2 \times 10^{17}}{E_{e}^{2}}\right)^{-1}, \mathrm{eV}-\mathrm{cm}^{2}$ 
The second equation is an expression of energy conservation applied at the receding pellet surface, whereby. the incident energy flux is equated to the rate of evaporative cooling

$$
\frac{\mathrm{dr}}{\mathrm{dt}}=-3.7 \times 10^{-14} \mathrm{n}_{e^{\mathrm{E}}}^{\mathrm{E} / 2} \mathrm{E}_{e_{0}}^{\mathrm{cm} / \mathrm{sec}} .
$$

Together, these expressions uniquely determine the unknown energy $\mathrm{E}_{\mathrm{e}_{0}}$ ' the pellet surface recession rate $d r_{0} / d t$, and hence the particle source rate. They are solved numerically for a pellet of a given initial size traversing a nonhomogeneous plasma at some specified speed and trajectory. The essential elements of the physical model that lead logically to the derivation of Eqs. (3.1 and 3.2) are stated below.

(1) The incident flux of electrons at the pellet surface is calculated from an assumed Maxwellian distribution of temperature $\mathrm{T}_{e}$.

(2) Elastic scattering of electrons and/or attenuation of the flux due to electrical charging of the pellet is neglected. This is a conservative assumption since the inclusion of a potential would reduce the heat flux to the pellet, thus allowing greater penetration of the pellet into the plasma.

(3) The surface of the pellet responds to the heat input by evaporation of molecular hydrogen only.

(4) The resultant cloud of neutral hydrogen that surrounds the pellet surface is quasi-steady in nature.

(5) The physics of the expansion of the shielding cloud is determined by volumetric heat due to streaming primary electrons undergoing inelastic collisions with the molecular hydrogen.

For justifiration and further explanation of these points, the reader is referred to Refs. 3 and 18 . It is assumed in this study that the neutrals given off by the pellet are ionized in the outer regions of the cloud by energetic electrons before encountering fast ions. This essentially precludes, in the conceptual sense, the formation of high 
energy charge-exchange neutrals. Examination of this assumption will be the subject of future pellet injection experiments at Oak Ridge National Laboratory (ORNL).

\subsection{ANALYTIC SOLUTIONS}

The idiosyncrasies of the model and clarification of some of the points mentioned above follow from consideration of a specific example for typical plasma conditions. In Fig. 1, Eqs. (3.1 and 3.2) are plotted as a function of the unknown $\mathrm{E}_{\mathrm{e}_{0}}$ for the conditions $\mathrm{r}_{0}=2 \mathrm{~mm}, \mathrm{n}_{\mathrm{e}}=$ $10^{14} / \mathrm{cm}^{3}$, and $\mathrm{E}_{\mathrm{e}}=10 \mathrm{keV}$. The most important conclusion to be drawn $1 \mathrm{~s}$ that the intersection of the two curves which represent the solution for $\mathrm{dr}_{0} / \mathrm{dt}$ occurs at a low value of $\mathrm{E}_{\mathrm{e}_{0}}$ where the curve representing $\mathrm{Eq}$. (3.1) is uniformly flat. This means essentially that Eq. (3.2) need not be considered and that the solution for $\mathrm{dr} / \mathrm{dt}$ is represented by F. . (3,1) alone, evaluated at any value of $\mathrm{E}_{e_{0}}$ between $1 \mathrm{eV}$ and $1 \mathrm{keV}$. It can be readily verified that even large changes in the electron flux $\left(\propto_{e} \sqrt{E_{e}}\right)$, the plasma density, and the pellet radius will not alter this fact appreciably. In particular, the attenuation of the electron flux brought about by elastic scattering and/or negative charging of the pellet will not signiflcancly reduce the ablation rate. The explanation for this behavior is straightforward; the material expelled by the surface is so effective at absorbing the energy of primary electrons that additional reductions in the Incident heat flux by attenuation of electron current is not neceseary.

The above conslderations lead at once to a simplified, but accurate, pellet lifetime scaling law that is useful. for illustrating the sensitivily of the model. to the physical parameters. Taking $10 \mathrm{cV}$ as a typical value for $\mathrm{E}_{\mathrm{e}}$, $\mathrm{Eq}$. (3.1) reduces to

$$
\frac{d r_{o}}{d t}=-\frac{1.75 \times 10^{-2}}{r_{o}^{2 / 3}} \frac{n_{e}^{1 / 3}}{M^{1 / 3}} E_{e}^{1 / 6}\left[L\left(\frac{E_{e}}{2}\right)\right]^{1 / 3}\left(E_{e}+8.5 \times 10^{-4} E_{e}^{2}-\frac{850}{E_{e}}+75\right) .
$$




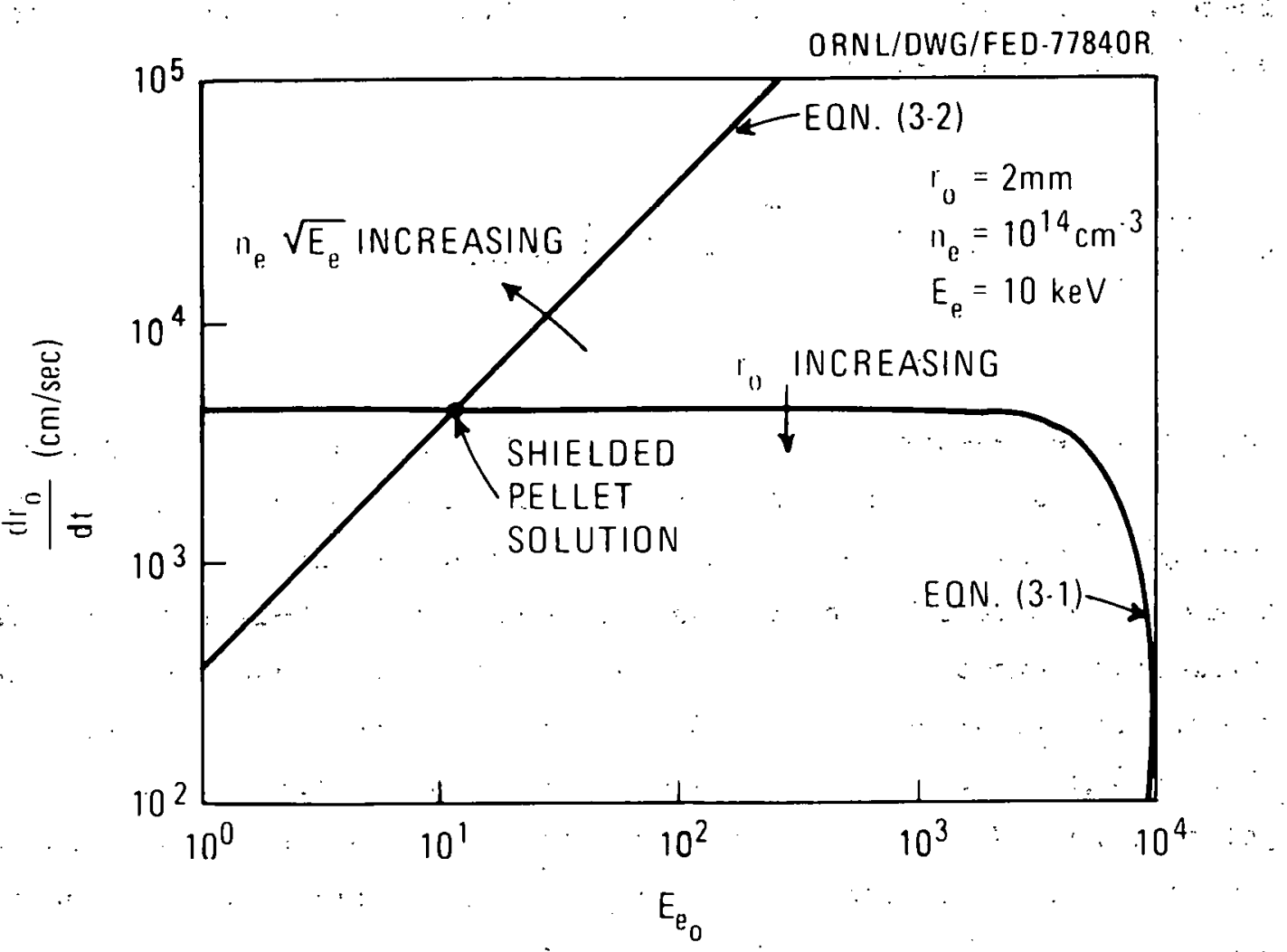

Fig: 1. The simultaneous solution of Eq:. (3:1) (which gives the rate at which the pellet must supply particles to the shield to reduce the incident electron energy from $\mathrm{E}_{\mathrm{e}}$ to $\mathrm{E}_{\mathrm{e}_{\mathrm{o}}}$ ) and $\mathrm{Eq} \cdot(3.2)$ (which represents conservation of energy at the pellet surface) gives the shielded peliet solution. 
Equation (3.3) can be solved directly for the pellet lifetime, given the temporal behavior of the plasma temperature and density. Alternatively one could perform the integration to determine how far a pellet of a given size and velocity would penetrate into a plasma along a specified trajectory. The result of such a calculation for plasmas whose density and temperature vary as $n_{e} \sim 1-(r / a)^{2}, T_{e} \sim n_{e}^{2}$ is given by Milora and Foster. ${ }^{3}$ The speed, $U$, required to penetrate a plasma to a fractional depth $(\ell / a)$. is given by

$\mathrm{U} \approx \frac{4.03 \times 10^{-8} \mathrm{a}<\mathrm{n}^{>1 / 3}<\mathrm{T} \mathrm{e}^{>1.7}}{\mathrm{M}^{1 / 3}\left(\mathrm{I}_{\mathrm{o}}\right)^{5 / 3}}\left(\frac{l}{-}\right)^{3} \mathrm{~m} / \mathrm{sec}$,

where $l / a$ represents the fractional penetration dopth to the plasuld center, $r_{0}$ is the initial pellet size, and the plasma parameters are average values. Because of the strong $\ell / a$ dependence, velocity projections to penetrate beyond the halfway point in a TNS-like plasma are commonly in excess of $5 \times 10^{5} \mathrm{~cm} / \mathrm{sec}^{3}$ From the standpoint of relieving the technological burden placed on fueling devices, edge fueling is obviously attractive. The only other real alternative is to increase pellet size. Whenever possible, pellets should be injected at the maximum size which the plasma will allow if deep penetration is found to be necessary. It should also be noted that, since the parameter $\ell / a$ in Eq. (3.4) is the fractional distance to the plasma center, any outward shifting of the plasma by finite $\beta$ effects would be beneficial.

Fueling will, of course, alter the plasma profile beyond the simple shapes used to derive Eq. (3.4), and this is the subject of the work reported in Sect. 6 .

\section{FUELING PROFILE EFFECTS ON IGNITED PLASMA EQUILIBRIA}

Many previous studies of tokamak reactor systems have used zerndimensional analyses (e.g., see Ref. 25) which implicitly assume some cold particle fueling profile. Even the studies which used one-dimensional 
analyses (e.g., see.Ref. 26) have for the most part assumed.a particle source profile and have not investigated the effects of tailoring the source profile. Some fueling profile effects have been noted in past one-dimensional studies but have not been published. 27 Much of the reason for lack of work in this area stems from the need to have a physical model for particle fueling. A pellet ablation model has been developed 8 and coded $^{3}$ at ORNL, and the results of using this code are presented in Sect. 6 of this report. Preliminary to using this model, it was decided to study trends in tokamak behavior by using an assumed cold fuel deposition profile and adjusting the profile parametrically. The profile chosen for this work has the form

$S_{p}(r)=\left\{\begin{array}{rl}S_{p}(a)\left[1-\frac{(a-r)}{r_{p}}\right] & r>\left(a-r_{p}\right) \\ 0 & r<\left(a-r_{p}\right),\end{array}\right.$

where $a$ is the plasma minor radius and $r_{p}$ is a penetration length measured from the plasma edge. This model allows one to go from a flat fueling. profile $\left(r_{p} \rightarrow \infty\right)$ to an edge (neutral recycling type) fueling profile $\left(r_{p} \ll a\right)$ by varying only one parameter. It will be shown in Sect. 6 that the self-consistent pellet fueling profiles appear to be more rectangular than triangular in shape, but this does not affect the qualitative results given in this section. The absolute values of the numbers presented should not be weighed too heavily since transport coefflctents for both present-day and particularly future reactor grade plasmas are not accurately known.

In this section of the report the effects of the cold particle source profile on equilibrium plasma profiles are examined. The complete divertor model proposed by Mense and Emmert ${ }^{6}$ is used in the region $a<r<a_{w}$, where $a_{w}$ is the first wall radius. It is assumed. that the divertor absorbs all plasma entering it and that no gas, cold plasma, or impurities recycle from the divertor chamber. This is no doubt unrealistic, but it provides a base line calculation for this initial fueling analysis. It has been shown ${ }^{6}$ that small amounts of divertor recycling 
(say $10 \%$ of collected flux) have a small ( $<10 \%$ ) effect on plasma edge densities and impurity screening efficiencies.

As was indicated in Sect. 2, the trapped particle modes are assumed as the predominant driving mechanism for plasma particle and energy transport. For the work presented in this section, the diffusion coefficients and thermal conductivities are evaluated with local values of the density and temperature gradients. In order to save computer time, a thermally stable (ignited) equilibrium using a flat fueling profile was first found and then successive equilibria were generated by gradually changing the fueling profile. At each $r_{p}$ value the minimum average plasma density which still maintains the reactor in an ignited equilibrium is snught.

The results of this search are shown in the following series of figures. Figure 2 shows the effect of going to more shallow fueling profiles while keeping the average density fixed. The more shallow the fueling, the "flatter" the density profile becomes. In turn, the temperature profiles become more peaked and the fusion power increases. Figure 3 shows the effect which the cold fueling profile can have on the thermal fusion power. Shallower fueling decreases the thermal losses of the trapped particle modes by flattening the density profile. For a given volume-averaged density, the plasma temperature rises as the density profile is tlattened and thermal fusions increase. For a given fueling profile, the average density can be reduced until the plasma quenches. This minimum density is lower for shallower fueling because the thermal losses are lower. In general, lower density operation is found to be "inaccessible" with deep fueling, and the associated parameter region is marked in Fig. 3.

F1gure 4 shows the effect of two different fueling profiles on the density and temperature profiles. The average density in each case is reduced to 1es lowest possible value while still maintaining an ignited plasma. One can see clearly that $\nabla_{n}$ is markedly reduced by shallower. fueling, and in fact the temperature is kept low over the outer portions of the plasma due to the cold fueling. "The temperature peaking at the plasma center results from two effects. First, the trapped ion mode has been effectively shut off due to the flattening of $\nabla \mathrm{n}$. This causes reduction in $x_{i}$, which can support a large temperature gradient. 

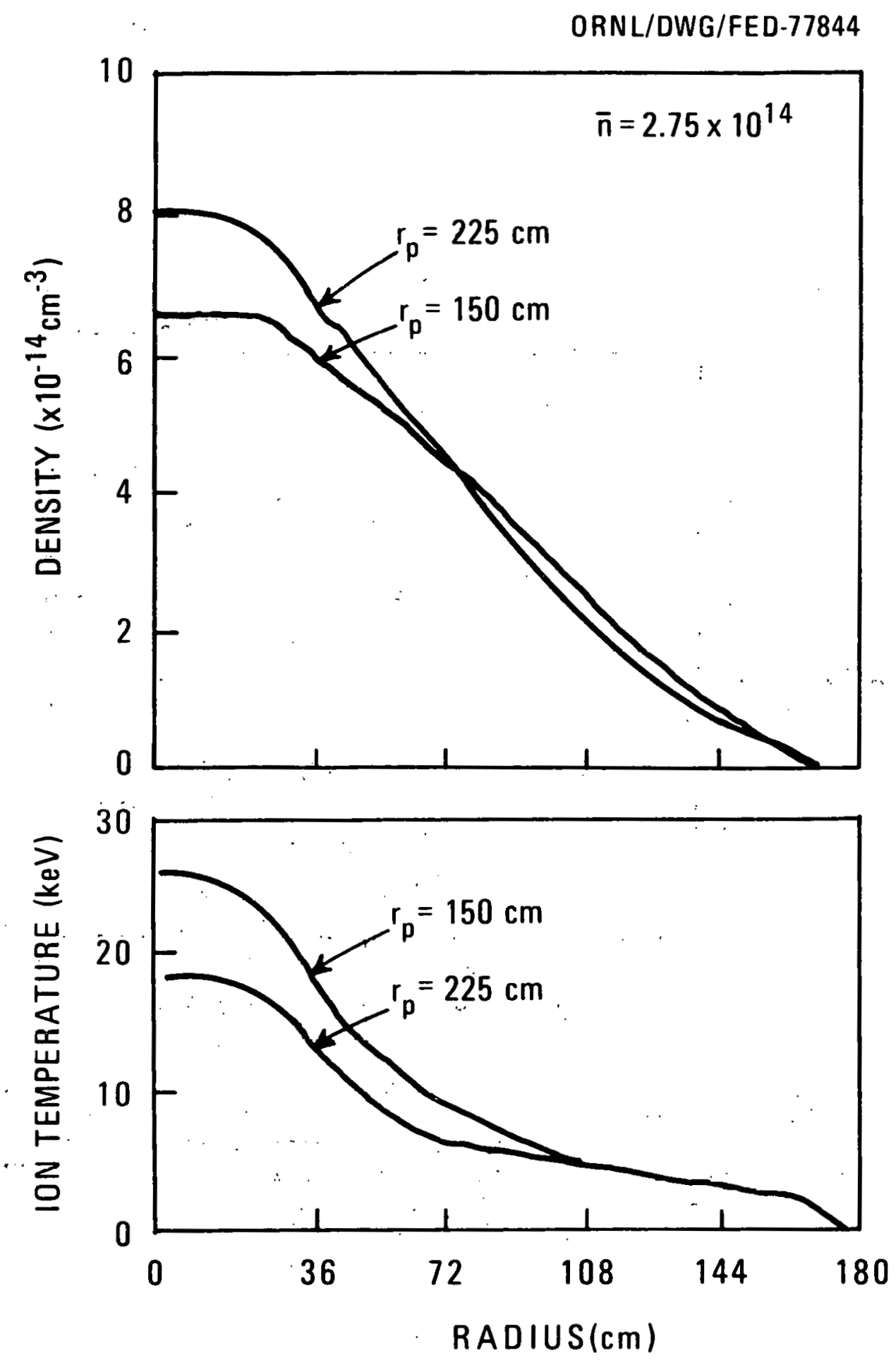

Fig. 2. Comparison of density and temperature profiles for two particle fueling depths with the same volume-averaged density. Shallower fueling penetration leads to a flatter density profile but a wiotie peaked tempcrature profile. 


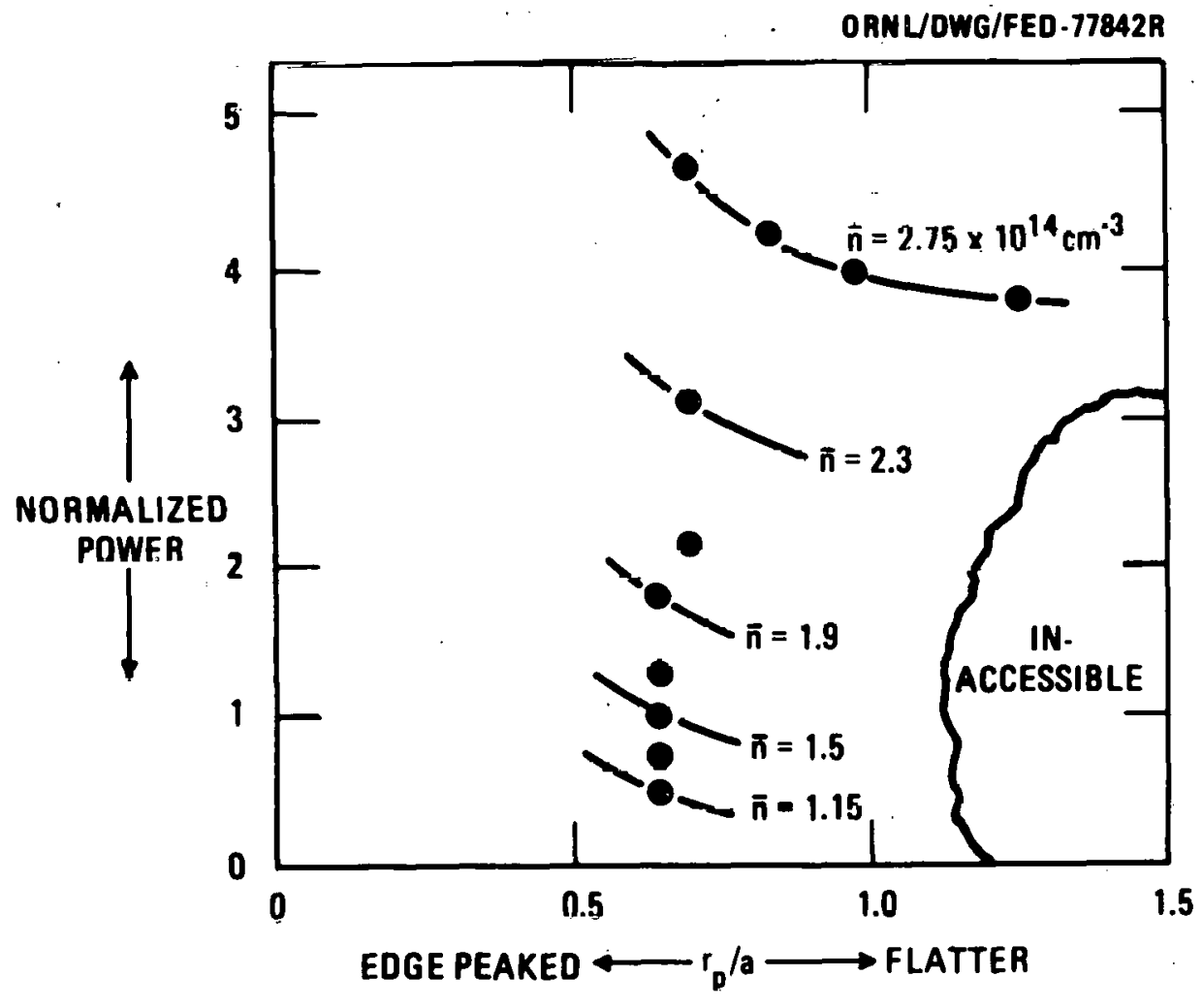

F1g. 3. l'he tusion power density at thermal equilibrium decreases with deeper fuel penetration because of enhanced energy losses associated with trapped particle microinstabllities. Deep fueling (flatter particie snurce prefile) oan lead to large enough energy losses to quench low density plasmas." 'l'his is nuted as the inaccessible regime. 
ORNL/DWG/FED-77843
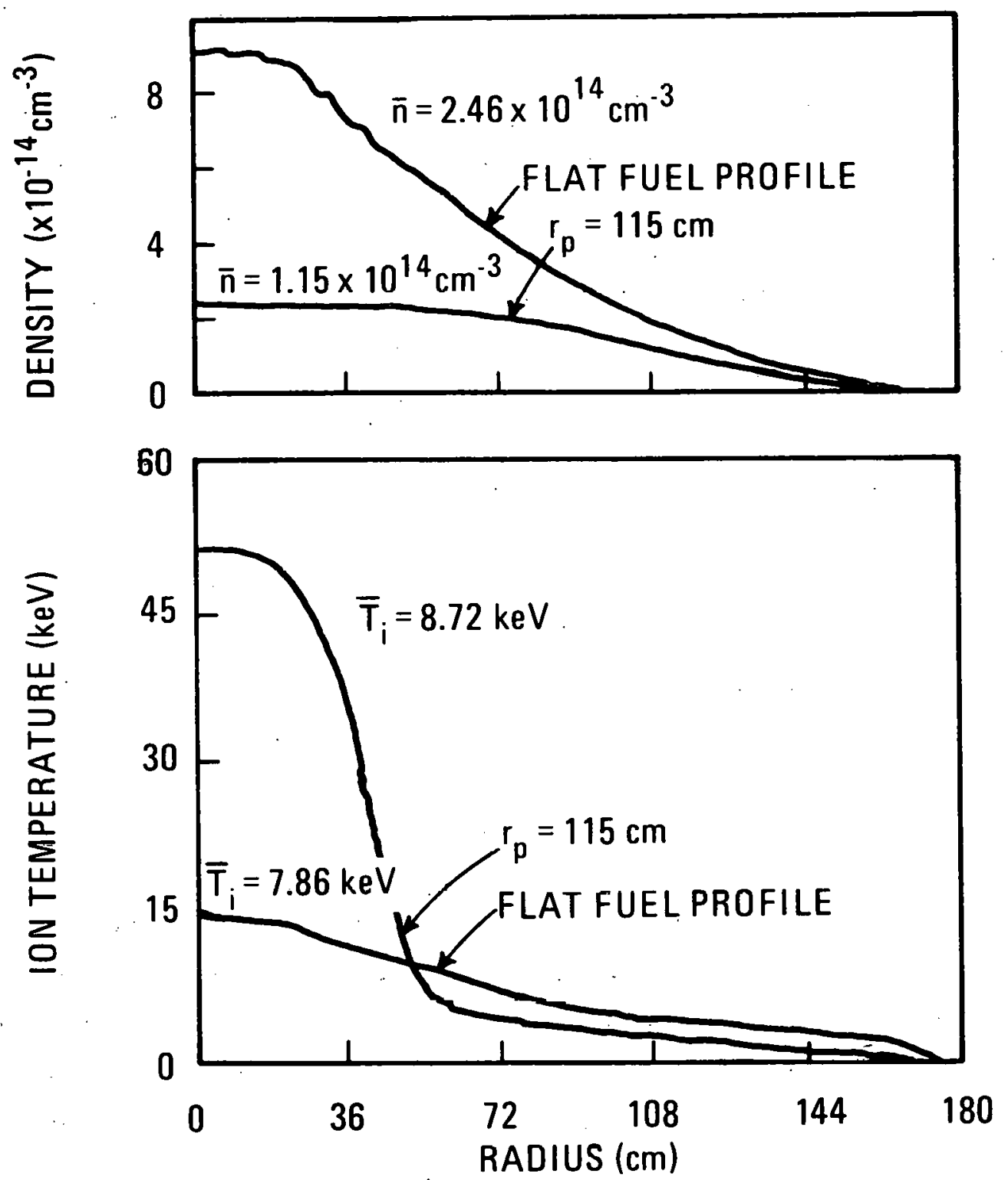

Fig. 4. Comparison of density and temperature profiles for two particle fueling depths. The average density in each case is the lowest value for which an ignited plasma can be sustained with a trapped particle transport model. 
Secondly, the peaking is somewhat enhanced due to the neglect of finite alpha particle orbit effects which smooth out the heating profile somewhat. These effects can become important in an absolute sense, but the same qualitative behavior would be observed even including alpha orbit effects.

Another point to be made concerning the general behavior of more shallow fueling profiles is the reduced fueling requirement; i.e., the particle (and energy) confinement times can be made to increase by making the fueling profile more shallow. This is illustrated in Fig. 5, where there is a greater than ten-fold reduction in the required fueling rate for the case where $r_{p}=115 \mathrm{~cm}$ over the fllat profille rase. Figure 6 shows the effect of fuel penetration and plasma density on the particle confinement time. In general, as the particle source penetration is reduced, the particle confinement time is expected to decreace because the particles are deposited closer to the plasma edge and are more likely to diffuse from the system. However, this effect is overshadowed by the reduction in the diffusion coefficient for shallower fuel penetration because of the trapped particle transport model. $\left[D \sim(\nabla n)^{2}\right]$, and the particle confinement time actually increases with lower fuel penetration in the range of $\mathrm{r}_{\mathrm{p}}$ /a considered here. In Sect. 5 very shallow fueling profiles $\left(r_{p} / a<U .5\right)$ are shown to reverse this trend for the trapped particle mules. IL shuuld also be noted in f'ig. 6 that higher density reduces the equilibrium particle confinement time. Higher density increases the collisionality and should reduce the trapped particle transport rnefficients, but higher density also results in an increase in fusion power and a stronger increase in temperature. This results in a net decrease in collisionality as the density is raised and a corresponding decrease in the particle confinement time.

Further details of the data used for the results of this section are contained in Appendix B. Also included are cases for which the amplitude of the trapped particle transport coefficients is arbitrarily decreased by up to an order of magnitude.

The $r_{p}$ values used for this phase of the study were never decreased beyond $115 \mathrm{~cm}$ due to the occurrence of numerical instability problems. 
ORNL/DWG/FED-77845
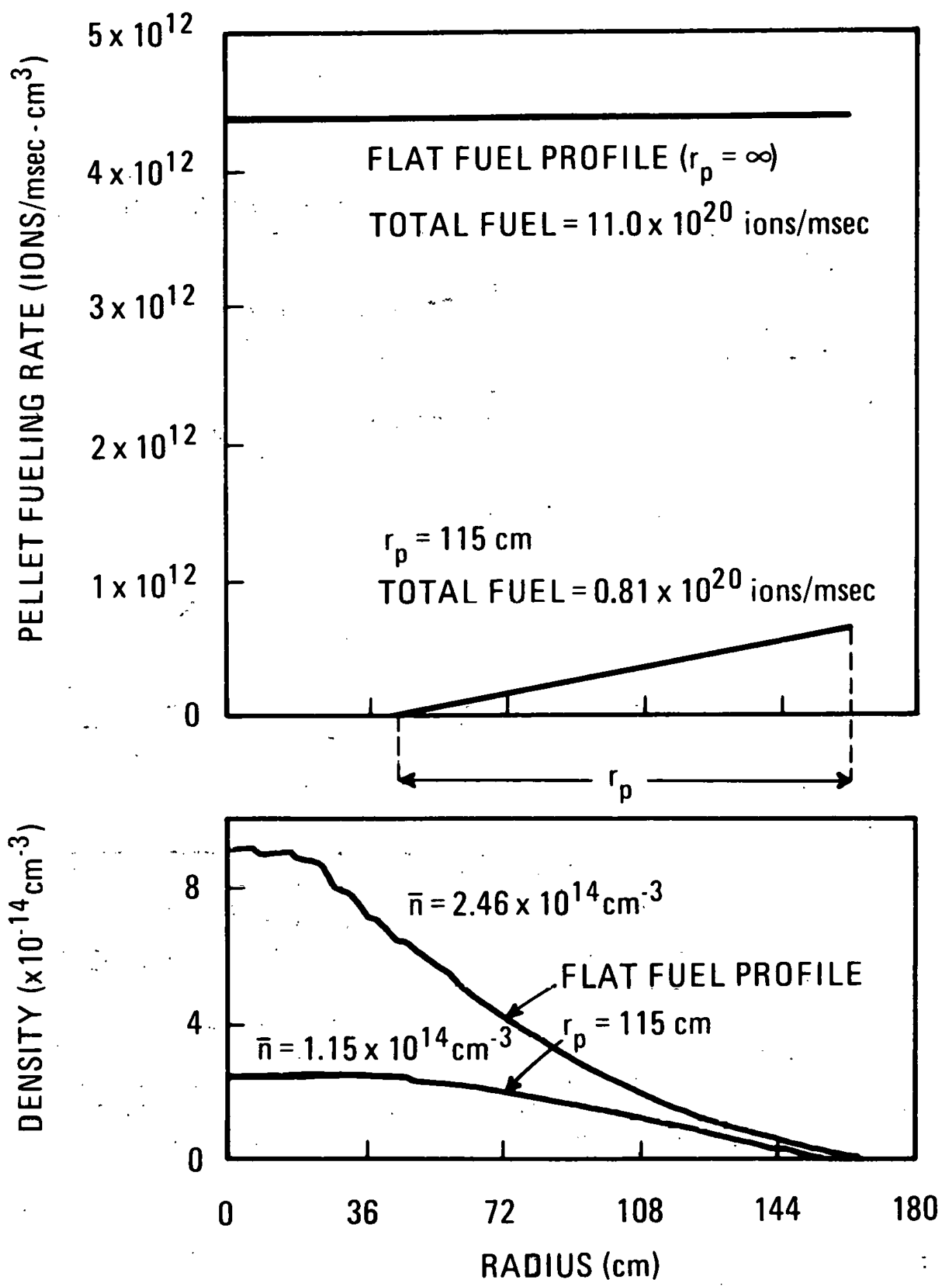

Fig. 5. Particle fueling requirements can be dramatically reduced by moderately peaking the particle source at the plasma edge if density gradient-driven trapped particle modes are : responsiblc for the diffusion. 


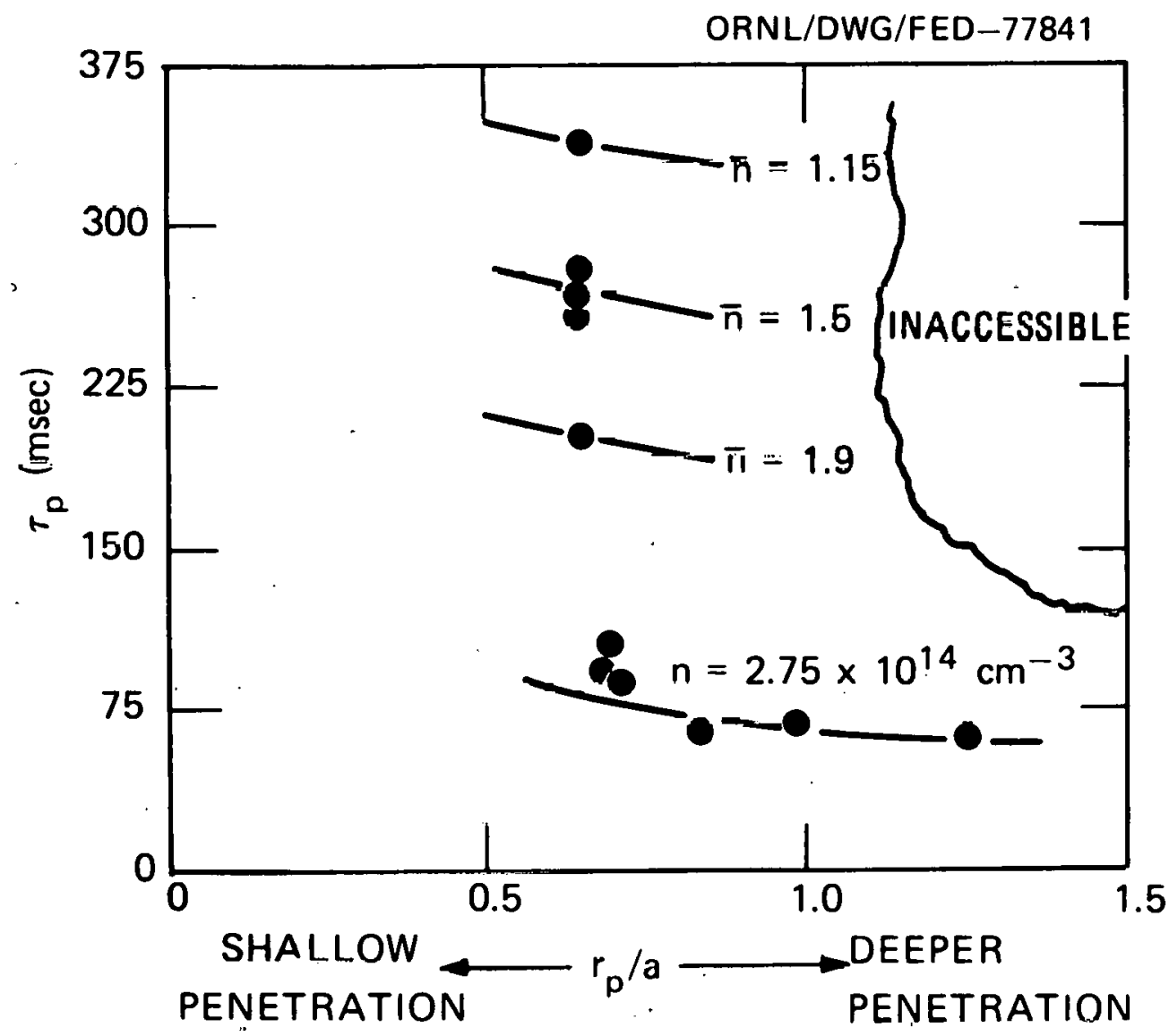

F1g. 6. The particle continement time increases for shallow penetration due to a decrease in the density gradientdriven trapped particle modes.. Higher densities increase the temperature of the thermal equilibrium, decrease the collisionality, and thus increase the trapped particle losses. 
As $r_{p}$ was reduced to values in the range of 75 to $50 \mathrm{~cm}$, numerical oscillations appeared in the electron temperature spatial profile. These were due to the sensitivity of the trapped particle mode coefficients to the density gradient, temperature gradient, and shear parameter (see Sect. 2). Since $\nabla n \sim 0$ for $r \lesssim\left(a-r_{p}\right)$, the modes are very sensitive to local gradient changes which occur when large timesteps are taken. It should also be noted that the transport coefficients shown in WASH$1295^{1}$ were derived assuming $n \equiv \mathrm{d} \ln \mathrm{T} / \mathrm{d} \ln \mathrm{n}$ is of order unity. In the central regions of the plasmas considered here, $|n|$ can easily be as large as 20 to 100 . Under these conditions the transport model becomes invalid. This is reiterated later in this report and indeed is an area needing more theoretical effort. In light of the above circumstances it seemed advisable not to push the calculations too far using this model. To summarize this section the following points are made:

(1) All numerical values quoted and graphs shown are results based on the use of full trapped particle modes (as stated in WASH-1295 ${ }^{1}$ ).

(2) Reducing the penetration depth of the fueling while maintaining the same fixed average density produces an increase in the fusion power production due to the peaking in the center of the temperature profiles.

(3) As the penetration depth is reduced, the average density required to maintain an ignited equilibrium decreases. This results in an increased confinement time, lower average temperatures, and lower fusion power production rates.

(4) For a given average density, "inaccessible" regions are found where the penetration is so deep that the increased trapped particle losses are so large that the plasma is unable to maintain ignition.

(5) The final point which must be emphasized is that further theoretical work is needed on transport models for the trapped particle modes in regimes where $\eta \equiv \mathrm{d} \ln \mathrm{T} / \mathrm{d} \ln \mathrm{n}>1$. 


\section{EFFECTS OF CONTINUOUS FUELING PROFILES \\ ON PLASMA TRANSPORT AND IGNITION}

Analytic particle fueling profiles linearly decreasing in intensity from the plasma edge are examined here to determine some basic relationships between fueling penetration depths and power required for ignition in a TNS plasma. A full neoclassical model for a multispecies plasma ${ }^{2}$ is used as a lower bound on the particle and energy transport in all cases. Application is made to a simplistic multiregime model for anomalous transport due to trapped particle microinstabilities (model discussed in Sect. 2). Attention is given to the way in which the particle fueling profile affects transport due to the trapped particle modes. Examined also is what the most important considerations should be in deriving transport models for the dissipative trapped particle modes under the conditions of edge fueling.

The plasma parameters considered here are given in Table 1. Two seconds of beam heating is used to determine whether the full-size plasma will ignite. For those cases which do ignite, the beam power is reduced to determine the minimum power for ignition of the plasma. The relative amounts of power required give an indication of the relative ease or difficulty of ignition. No attempt is made to optimize the ignition scenario to produce a minimum power requirement. Many additional factors which would affect such an optimization study are not included in this analysis (see Sect. 2). Lower density, smaller radius startup scenarios would likely provide an atractive alternative to the full density, fullsize startup used here. Lower beam power applied for longer times would also reduce minimal power requirements, but it would not reduce the total energy requirement.

The particle source profile used for all the following cases is one which linearly decreases in intensity from the plasma edge.

$$
\begin{array}{ll}
S_{p}(r)=0 & r<\left(a-r_{p}\right) \\
S_{p}(r)=S_{p}(a)\left(1-\frac{a-r}{r_{p}}\right) & r>\left(a-r_{p}\right),
\end{array}
$$


where $S_{p}(a)$ is the source intensity per unit volume at the plasma edge. When $r_{p}>a$, the source intensity does not vanish at the center of the plasma but is reduced by a factor of $\left(r_{p}-a\right) / r_{p}$ from the value at the edge. A feedback scheme is used on the source amplitude to maintain constant average density. A feedback response time of $10 \mathrm{msec}$ is used in all cases.

Average densities of $10^{14} \mathrm{~cm}^{-3}$ and $8 \times 10^{13} \mathrm{~cm}^{-3}$ are considered. The higher density cases require $200-\mathrm{keV}-\mathrm{D}^{\circ}$. beams for adequate penetration ( $12^{\circ}$ from perpendicular), while $150-\mathrm{keV}$ beams are sufficient for the lower density cases. However; $200-\mathrm{keV}$ beams are used here for both densities. Even though the alpha density balance is included in the analysis, the alphas do not account for more than a few percent of the total density. The evolution of the plasma is followed only long enough to determine if it has ignited, at which time a relatively low number of fusions has occurred.

Figure 7 indicates the effect of varying the particle source penetration depth and degree of anomalous transport on the minimum power for igniting a $\bar{n}=10^{14} \mathrm{~cm}^{-3}$ plasma. Model A includes trapped particle, pseudoclassical, and neoclassical transport as additive in their effects. For shallow penetration, $r_{p} / a \lesssim 0.5$, the power requirements are increased because of convective losses associated with fueling so close to the plasma edge. Decreasing penetration decreases the distance particles have in which to diffuse from the system. As the penetration is increased, the convective losses drop; conductive losses associated with the trapped particle modes extend deeper into the core of the plasima, and the puwer requirements rapidly increase. Fur $r_{p} / a \geq 0.7$ the plasma would not ignite. Figures 8 and 9 illustrate two particular cases. In Fig. 8 the ion temperature and density behavior are illustrated for $\mathrm{r}_{\mathrm{p}} / \mathrm{a}=0.5$ and $P_{i 1 j}=110 \mathrm{MW}$, which is near the minimum in the curve. The average ion temperature drops dramatically after the beams are turned off as the plasma heating profile shifts from the broad beam heated profile to the centrally peaked alpha heating profile. The drop in the average temperature is primarily due to a rapid cooling of the edge fueling region, where few alphas are produced. Several effects not in the model would be expected to broaden and smooth the temperature profile. Among them are 
ORNL/DWG/FED-77595

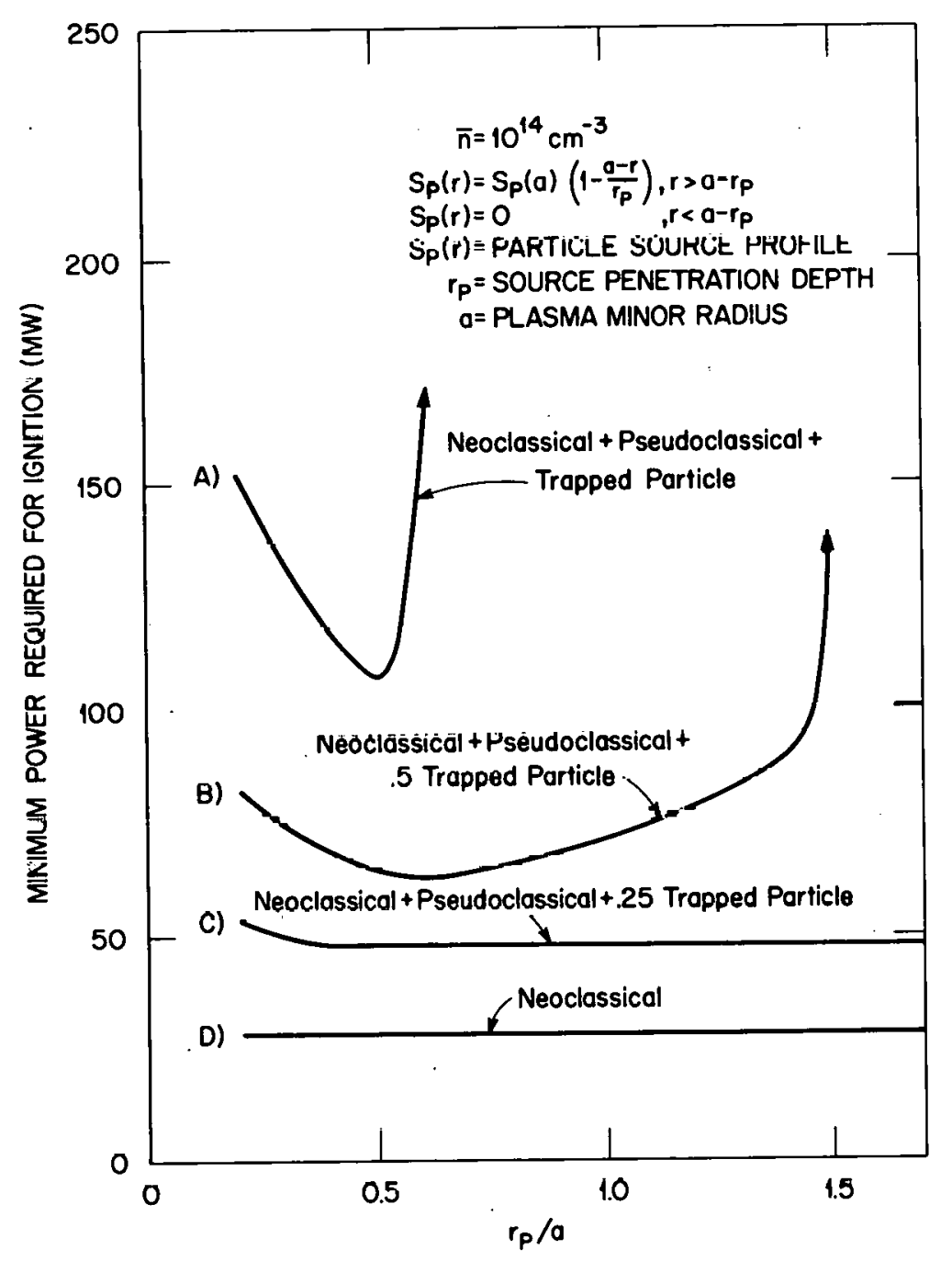

Fig. 7. The minimum neutral beam power $\left(200 \mathrm{keV} \mathrm{D}^{\circ}\right.$ applied for $\left.2 \mathrm{sec}\right)$ required to reach Ignttion in INS as a function of particle source penetration depth for several transport models. The volume-averaged density is $\bar{n}=10^{14} \mathrm{~cm}^{-3}$ for all cases. 

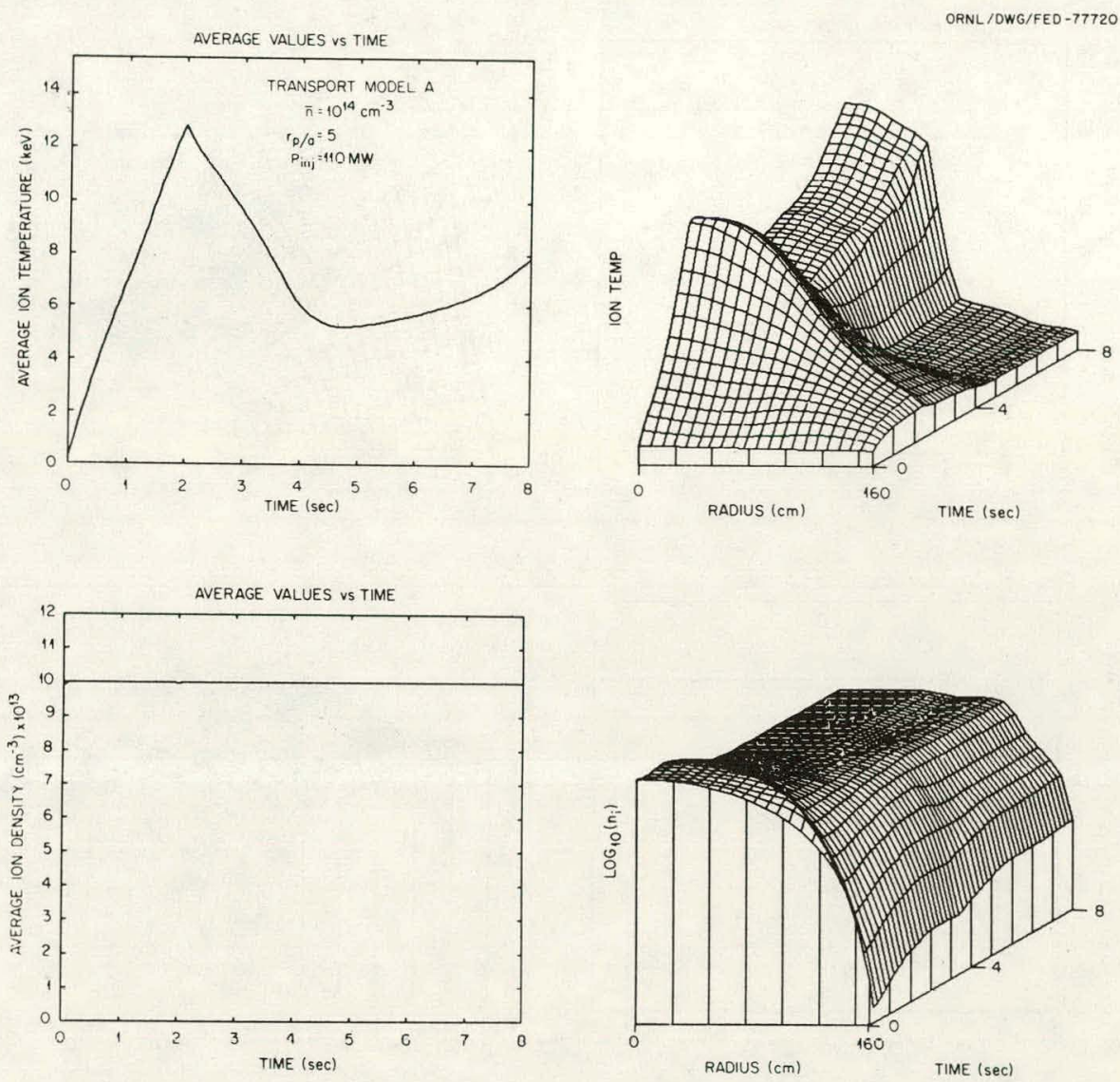

Fig. 8. Cold particle fueling keeps the temperature low in the edge region, $\mathrm{r} / \mathrm{a}>0.5$. The cemperature proftle shifts from the broad beam heating profile to a sharply defined hot central core (local alpha heating assumption) and cool edge region. Finite orbit effects and diffusion of fast alphas would tend to broaden and smooth the temperature profile. 

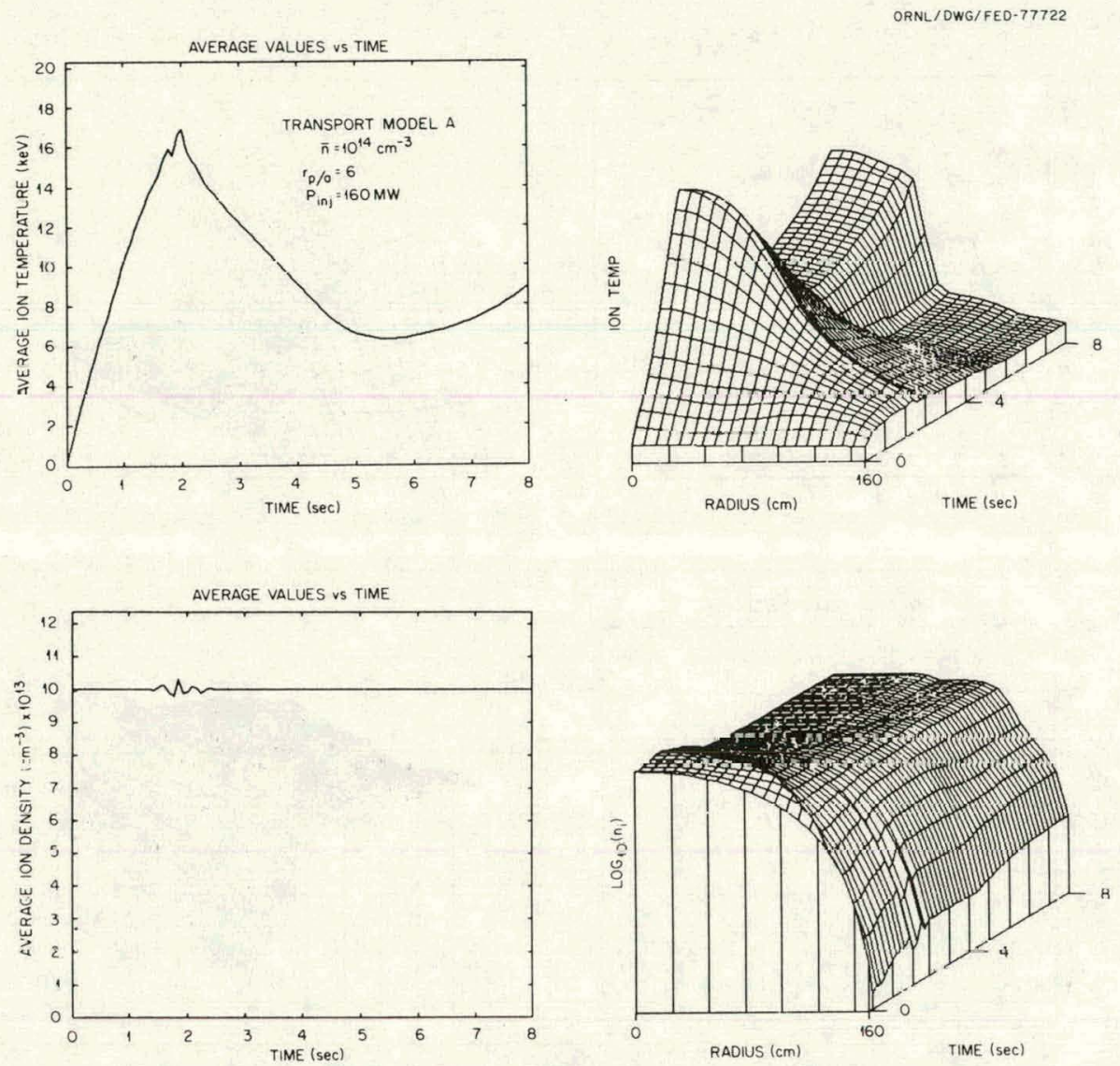

Fig. 9. The same case is illustrated here as in Fig. 8 but with slightly deeper fuel penetration. The ignited core and uniform density region is reduced in size, and trapped particle losses increase the required power to attain ignition. The fluctuations in $\overline{\mathrm{n}}$ are due to a shorter particle confinement time at high temperature with an assumed fixed particle feedback response time of $10 \mathrm{msec}$. 
finite orbit effects and diffusion of fast alphas. ${ }^{15}$ The density profile is nearly flat in the central region, where no fueling takes place, and hence the anomalous transport due to the trapped particle model used is very small. A model for residual modes would be more appropriate under these circumstances. Off-diagonal diffusion terms arising from anomalous transport could also modify the flat density central region (see Appendix A.) In Fig. 9 the fueling penetration is deeper $\left(r_{p} / a=0.6\right)$ and the hot core region is reduced in size. The fluctuations in the average density occur because the particle confinement time is reduced as the temperature rises, and the feedback response time of $10 \mathrm{msec}$ is inadequate.

In model $\mathrm{B}$ of Fig. 7 the magnitude of the trapped particle transport coefficients is reduced by a factor of two, while pseudoclassical and neoclassical expressions are retained. Convective losses at very low penetration depths still increase the power requirements as in model A. It takes much deeper fuel penetration to increase the density gradient and thus turn on the density gradient-driven trapped particle modes. Note that the minimum in the curve is only about a factor of two greater than pure neoclassical losses (model D). For $r_{p} / a>1.5\left[s_{p}(0)>s_{p}(a) / 3\right]$ no ignited cases were found. Figure 10 illustrates the behavior of the ion temperature and density for shallow penetration $\left(r_{p} / a=0.2\right)$. The cold edge region is very narrow, and the density is flat over almost the entire plasma. Oscillations in the average density due to low particle confinement time at high temperature are again evident.

The transport coefficients of the trapped particle modes are further reduced in model $\mathrm{C}$ of $\mathrm{Fig} .7$ by a factor of four from model A. A very slight increase in losses is noted at low penetration, while no increase is found at deeper penetration for the range considered. Figure 11 shows the plasma behavior for a deep penetration case $\left(r_{p} / a=1.6\right)$. The density and temperature profiles are both centrally peaked. The temperature profile smoothly transforms from the beam heating profile to the alpha heating profile. There is only a very small drop in the average temperature after the beams are turned off at $2 \mathrm{sec}$.

Model D of Fig. 7 is included as a base case with only neoclassical transport. The beams provide more than enough particles to fuel the plasma, and no additional fueling is required during the $8 \mathrm{sec}$ the plasma 

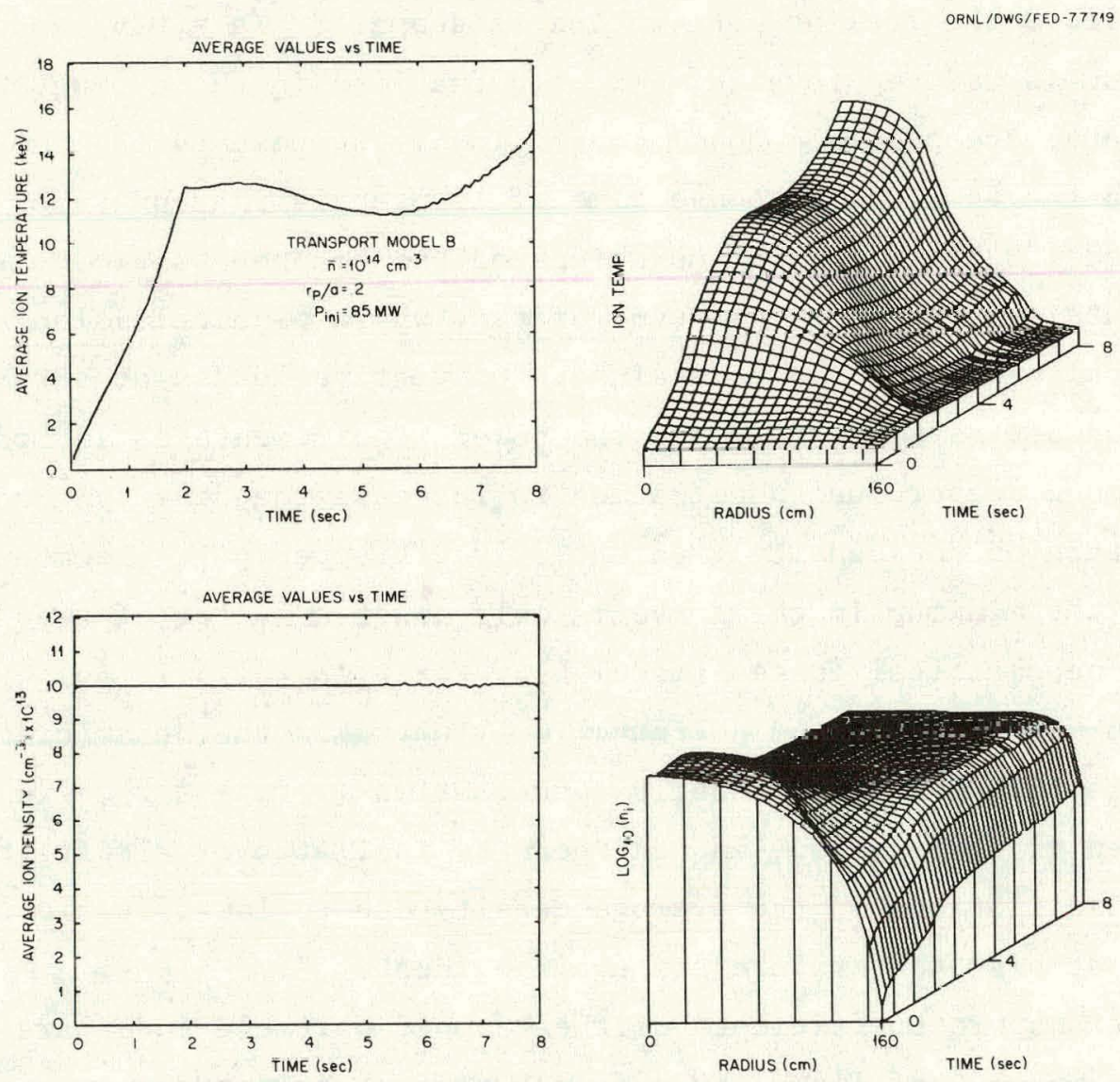

Fig. 10. With very shallow particle fueling the edge region becomes cooler and narrower. The uniform density core region is broader; therefore, pinch and Nernst terms in the particle diffusion model should become important. 

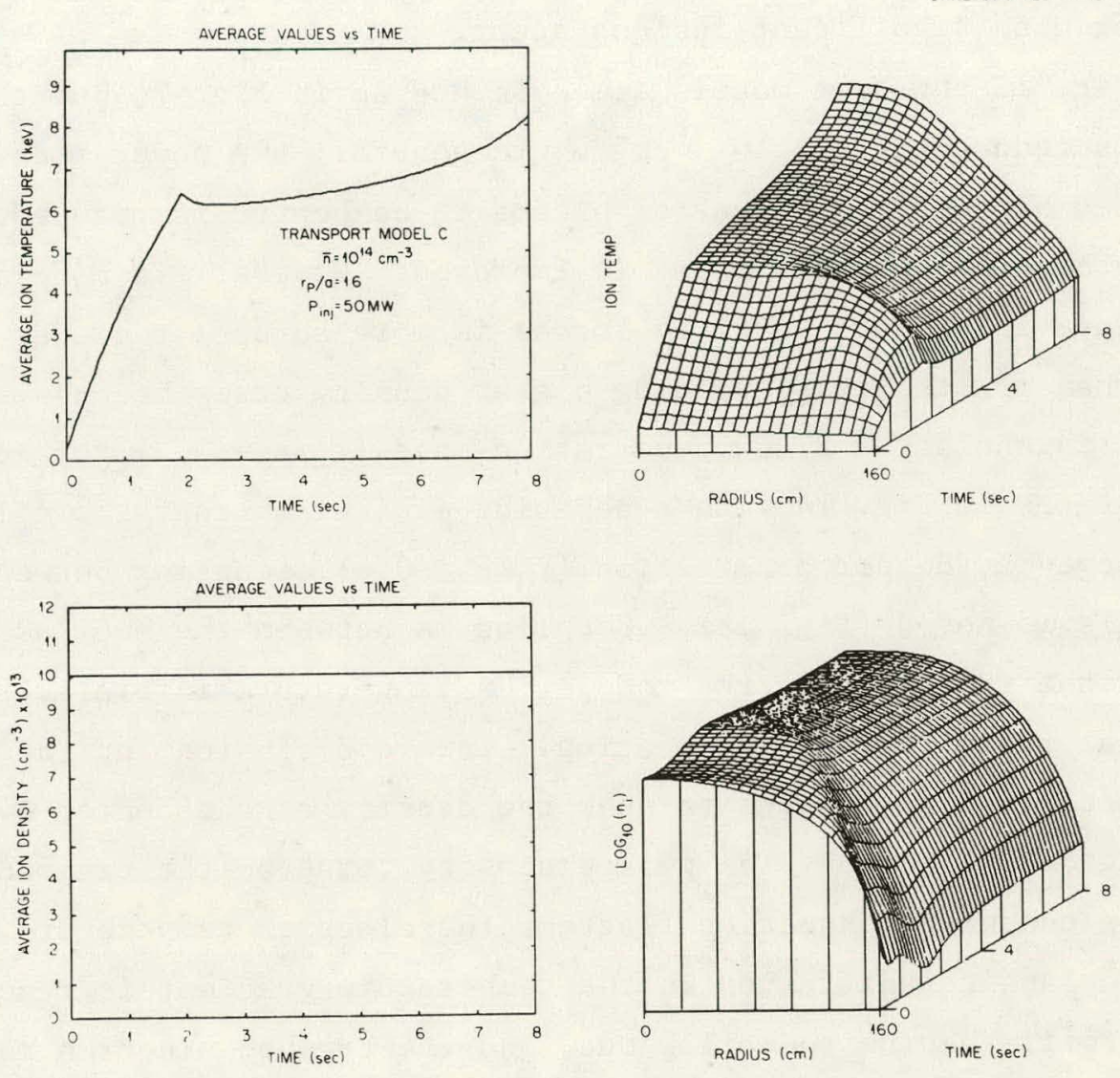

Fig. 11. Fueling occurs throughout the volume of the plasma but is still edge peaked. The density is centrally peaked and uniformly decreasing. The edge temperature is higher due to reduced cooling by the particle source. 
evolution is followed. Figure 12 illustrates this behavior. The density builds up during the $2 \mathrm{sec}$ the beam is on, then very slowly decays. The electron temperature profile is included here because it reflects the beam heating profile. With the very low neoclassical electron conductivity, the electron temperature profile follows the beam heating profile, slowly relaxes after the beams are turned off, and then begins to peak at the center when significant fusions occur.

In Fig. 13 the same models are examined as in Fig. 7, but the density is reduced to $8 \times 10^{13} \mathrm{~cm}^{-3}$. In general, the power requirements are reduced because the amount of plasma to be heated is reduced, and the penetration of the neutral beams is improved. However, in models $A$ and $R$ the increase in trapped particle losses is more abrupt at deeper penetration than for the corresponding higher density cases because of the lower collisionality. A low penetration case $\left(r_{p} / a=0.2\right)$ for model $B$ is illustrated in Fig. 14 with a behavior similar to that shown in Fig. 8 .

At somewhat deeper penetration $\left(r_{p} / a=0.6\right)$, coupling between transport models is noted (Fig. 15). Coupling is between the nenclassical Lerms and the trapped electron mode. The temperature initially rises in the central region from alpha heating. Tnward diffusion, up the clectrun temperature gradient, begins to peak the density at the center even though no fueling occurs there. The peaked density turns on the trapped electron mode. The enhanced conduction flattens the electron temperature profile. Inward neoclassical diffusion on the temperature gradient is reduced, the density profile begins to hollow out, and the trapped electron mode is turned off. Even though the model for the trapped electron mode can be seriously questioned, two important points can be made. First, the off-diagonal terms in the transport model can have significant impact on the transport behavior. Second, there may be coupling between kinetic models fur Lransport to produce temperature and/or density oscillations analogous to sawtooth oscillations (observed experimentally) but on a larger scale.

In model $\mathrm{C}$ there is no increase in convcctive losses al low penetration, while there is an increase at higher density. This may be partly due to better neutral beam penetration. With less beam energy deposited in the cold fueling region, the heating is more efficient. In 

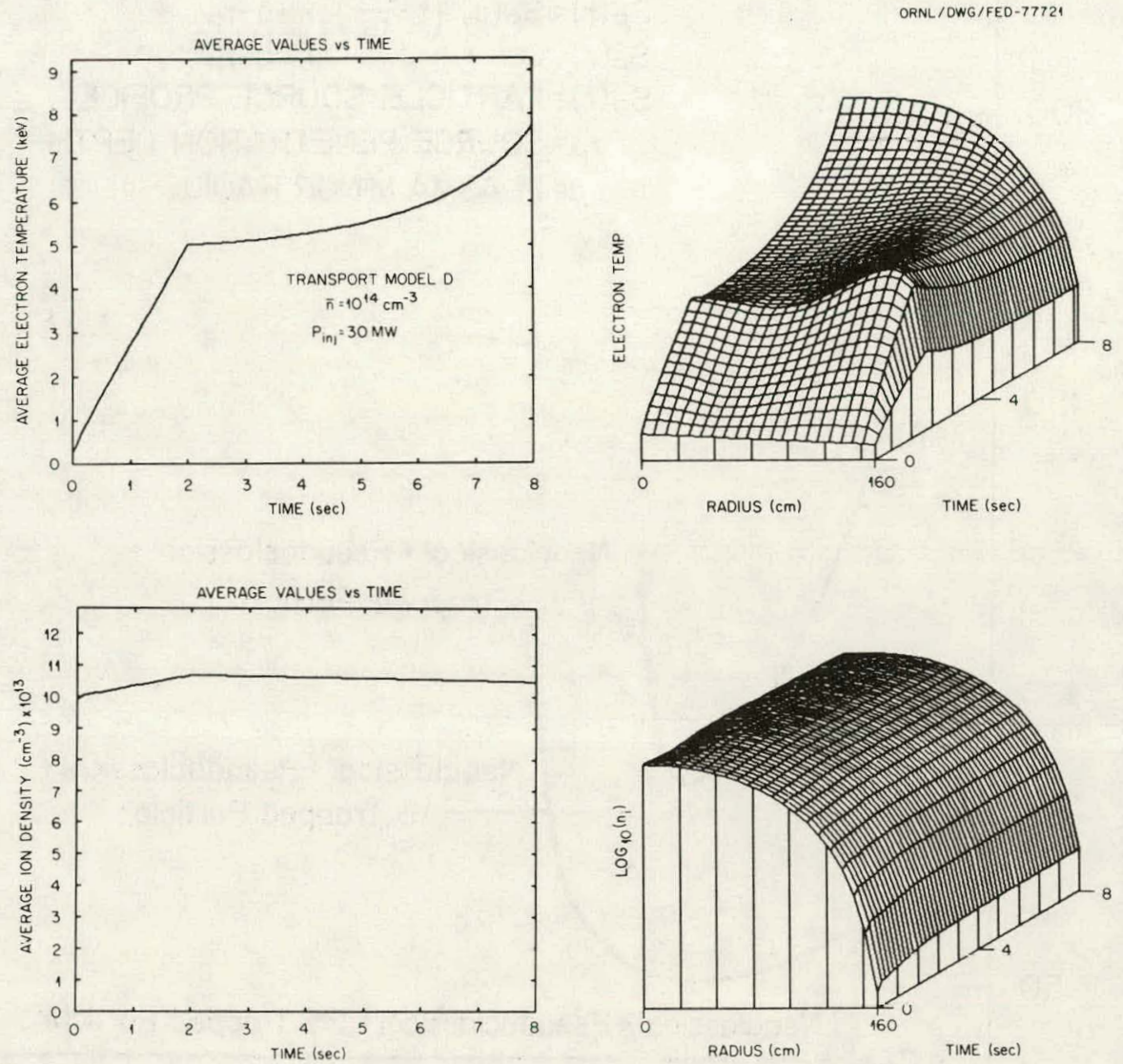

Fiy. 12. The neoclassical transport model gives very low diffusion losses. The source of particles from the neutral beams during the $2-$ sec beam heating phase increases the density, and no additional source of particles is required during the length of the simulation. 


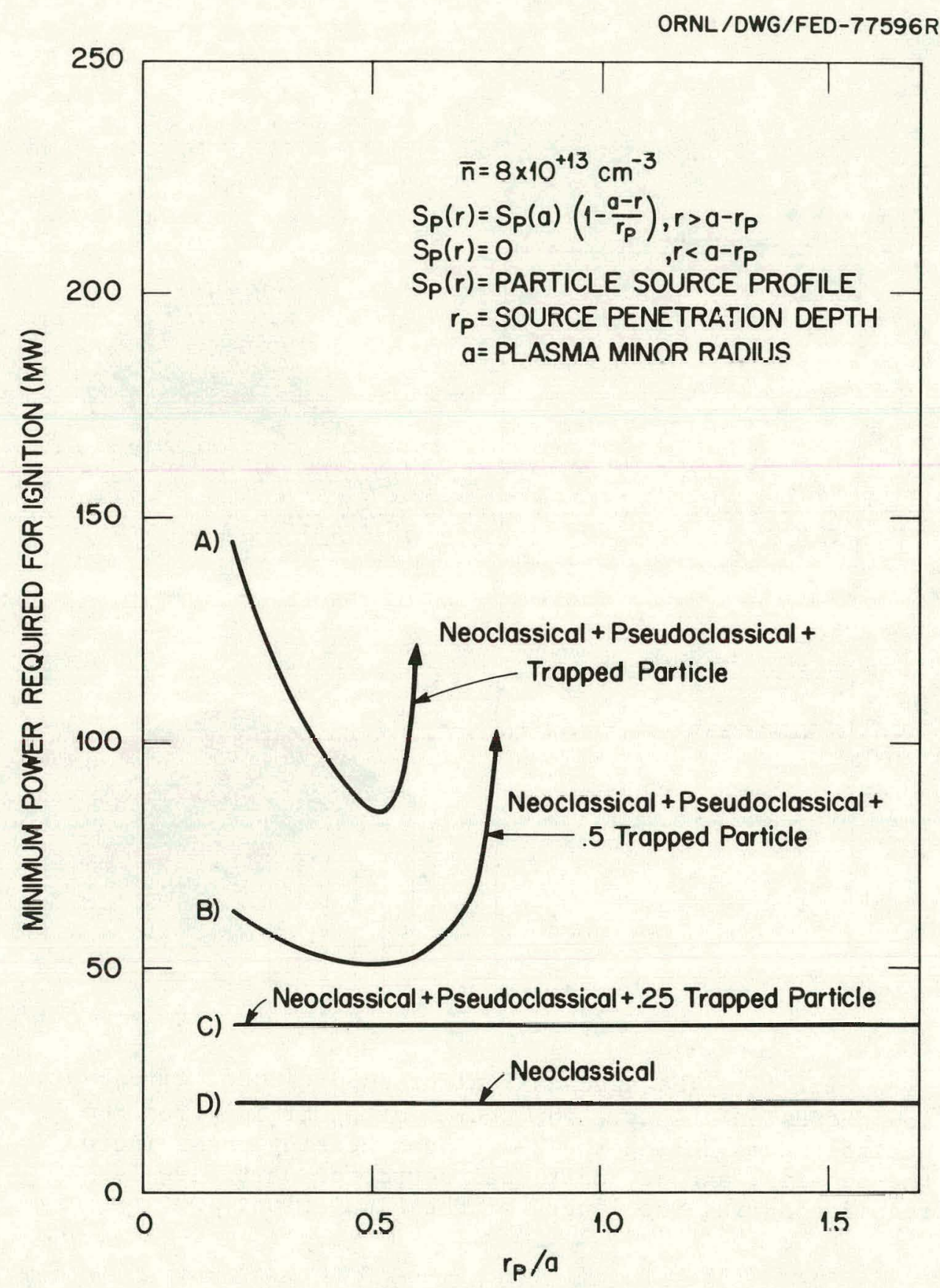

Fig. 13. The minimum neutral beam power $\left(200 \mathrm{keV} \mathrm{D}^{\circ}\right.$ applied for $2 \mathrm{sec}$ ) required to reach ignition in TNS as a function of particle source penetration depth for several transport models. The vulume-averaged density is $\overline{\mathrm{n}}=8 \times 10^{13} \mathrm{~cm}^{-3}$ for all cases. 

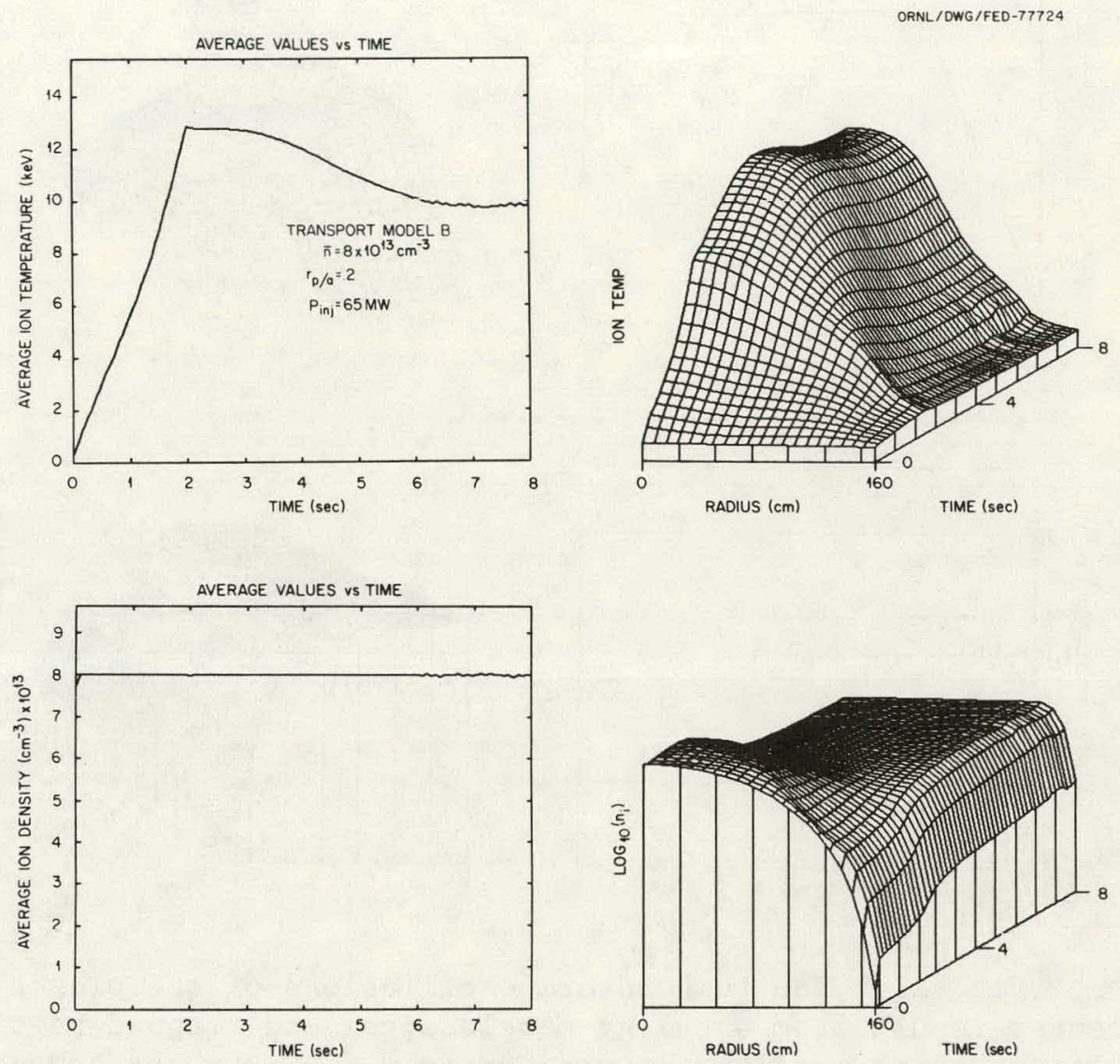

Fig. 14. The shallow penetration of the particle source leads to a more collisional, cooler edge region around a fairly uniform density core. The behavior is very similar to that shown in Fig. 10. 

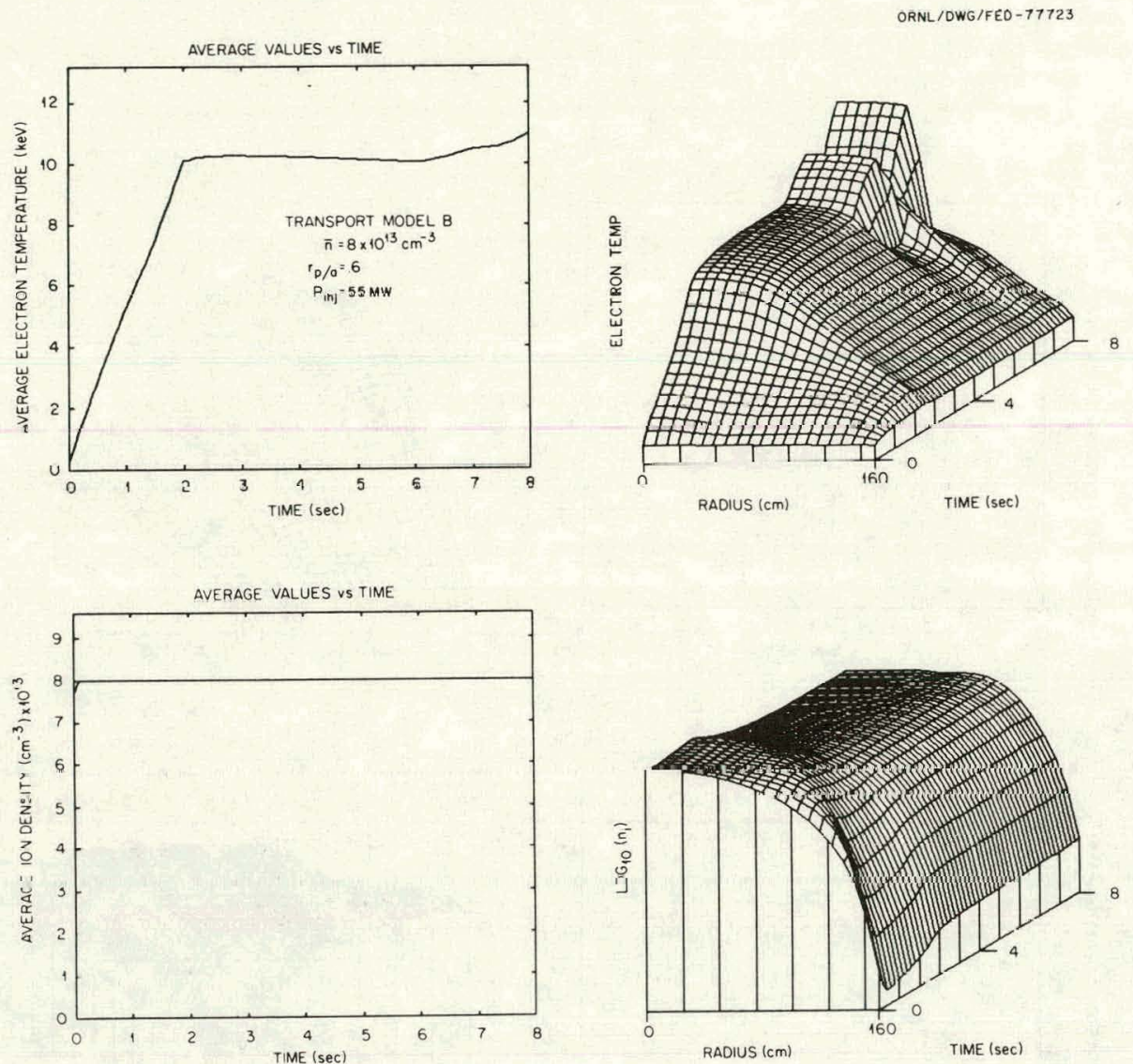

Fig. 15. The temperature oscillations of the plasma center result from coupling neoclassical and trapped particle transport models. Inward particle flow ups the temperature gradient (neoclassical Nernst term), peaks the density profile, and turns on the enhanced transport. The temperature protile broadens and inward Nernst diffusion is decreased. Fuel burnout begins to hollow the density profile and shut off the trapped particle transport. Local alpho heating begins to pcalc the tempcrature profile, and the entire process is repeated. Although many of the details of the physics can be questioned, the importance of coupled transport models becomes apparent. 
both models $\mathrm{C}$ and $\mathrm{D}$ the beam power requirements are reduced in proportion to the reduction in plasma density.

The effects of finite thermalization time for the fusion alphas were examined for several cases. Only very modest delays in the heating rates were observed, and the minimum power to ignite was not increased by more than a few percent. At even lower densities, however, the classical thermalization time would increase, and the effects would be more notable.

In summary, control of the fueling profile gives an added degree of control of the plasma behavior. For the trapped particle model investigated in this work, intermediate fueling penetration depths can reduce power losses and therefore relax power requirements for ignition. Deep fuel penetration may be neither required nor desirable. On the other hand, very shallow edge fueling seems to increase convective losses at the plasma edge and have a strong cooling effect. Much more theoretical work needs to be done on the trapped particle transport models under the conditions of edge fueling. A controllable particle source profile, such as may be provided with pellets, can shed more light on the diffusion process. With this incentive, initial investigations of the behavior of fluid particle and energy balances with a discrete pellet fueling source have been undertaken and are reported in the next section.

\section{PELLET INJECTION SIMULATION}

The inclusion of the pellet deposition model in the transport code is an important step toward a realistic treatment of a fueling model. In the following 'I'NS' calculations, pellets shot into the hot, dense plasma were found to ablate completely near the plasma edge (20-30\% penetration), thereby cooling the edge and raising the edge density.

The highly peaked edge source produced a uniform density central region in which trapped particle transport losses were reduced. The low edge temperature tends to reduce sputtering by charge-exchanged neutrals, but more work remains to be done in this area. Parameter variations involving pellet sizes and velocities have not been carried out here. Most cases used $2-\mathrm{mm}$ radius pellets injected at $10^{3} \mathrm{~m} / \mathrm{sec}$.

The discreteness of the pellets poses special problems. In particular, strong temperature and density gradients arise on a fast time scale, 
which taxes the transport theory for a fluid plasma. Local kinetic effects due to perturbation of the local distribution function by the cold pellets should be further investigated by experiment and theory to determine whether pellet sizes must be limited. Based on these investigations, however, the following observations can be made with some confidence:

(1) The alpha power generated by a reactor fueled with low velocity pellets is fairly steady, since the temperature and density fluctuations are confined to the edge region.

(2) The pressure profile fluctuates very little, since the stored energy is not changed much by the introduction of a pellet.

Figuse 16 sliuws a 100-msec seyment of the evolution of a cypical plasma density profile (a segment of a longer simulation). At about $75 \mathrm{msec}$, the plasma density has relaxed to a monotonically decreasing function of radius with an average density of $3 \times 10^{13} \mathrm{~cm}^{-3}$. At that time, a 3-mm-radius pellet is injected into the plasma at a velocity of $10^{3}$ $\mathrm{m} / \mathrm{sec}$. 'l'he pellet ablates almost instantaneously ( $<1 \mathrm{msec}$ ), leaving a spike in the plasma density near the edge. Figure 17 shows the corresponding effect on the ion temperature. Initially the edge temperature is depressed by the presence of cold plasma from the previous pellet. As the edge warms, the particles diffuse out of the edge region. At $75 \mathrm{msec}$ the temperature drops sharply due to the injection of another pellet. Except for a short interval, the edge temperature remains at $\sim 0.5-1.0 \mathrm{keV}$ despite the fact that the beam is on during this segment of the simulation. The edge temperature is relatively high due to the divertor-type boundary conditions, which neglect recycling of cold gas and exclude impurities. Figures 18 and 19 show a complete simulation of a typical TNS case using smaller pellets in a more dense plasma. Most of the detail of the discrete pellets is washed out in these figures, due to the fact that 177 pellets are injected during the $8 \mathrm{sec}$; only 45 profiles are used in the creation of these three-dimensional plots. A 2-mm-radius pellet is injected whenever the average density falls below $1.0 \times 10^{14} \mathrm{~cm}^{-3}$. The pellet is injected at $10^{3} \mathrm{~m} / \mathrm{sec}$. For the first $2 \mathrm{sec}, 8 \mathrm{MW}$ of $200-\mathrm{keV}$ neutral deuterium beam is applied. The simulation is terminated at $8 \mathrm{sec}$, at which time the 


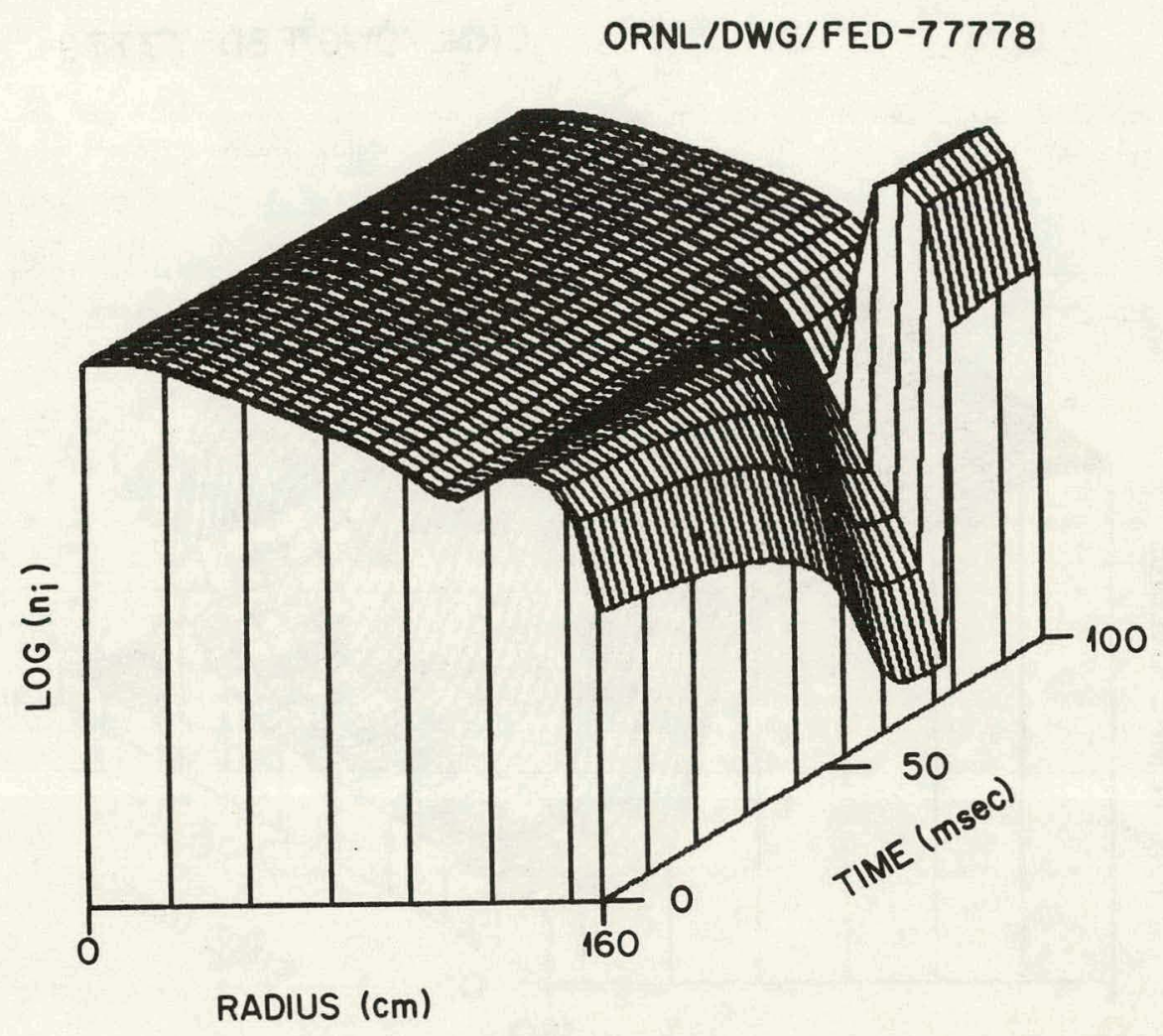

Fig. 16. Evolution of the density profile over $100 \mathrm{msec}$ for a large pellet, low plasma density case. A 3-mm-radius pellet is injected at $10^{3} \mathrm{~m} / \mathrm{sec}$ whenever the plasma density drops below $\overline{\mathrm{n}}=3 \times 10^{13} \mathrm{~cm}^{-3}$. Initially there is a bump in the density at the edge from a previous pellet; the bump decays away by diffusion until another pellet is injected at about $75 \mathrm{msec}$. Neutral beams are on during this phase of the calculation and account for the slight peaking of the plasma density. 


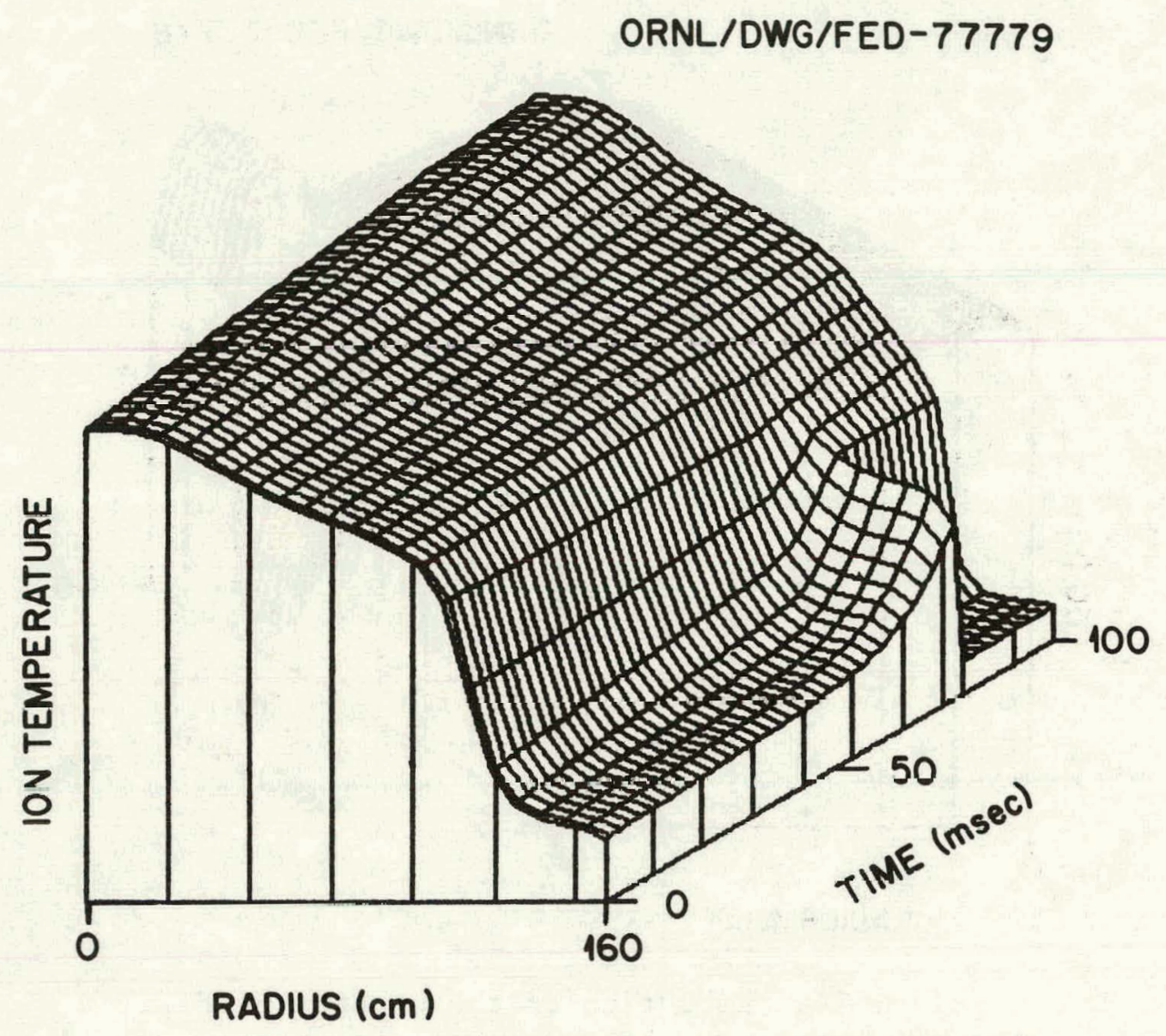

Fig. 17. Evolution of the ion temperature profile for the same segment of simulation shown in Fig. 16. The temperature at the edge of the plasma drops rapidly due to the cooling effect of the pellet. No significant increase in neutral beam attenuation was noted due to the pellet ablation spike. 


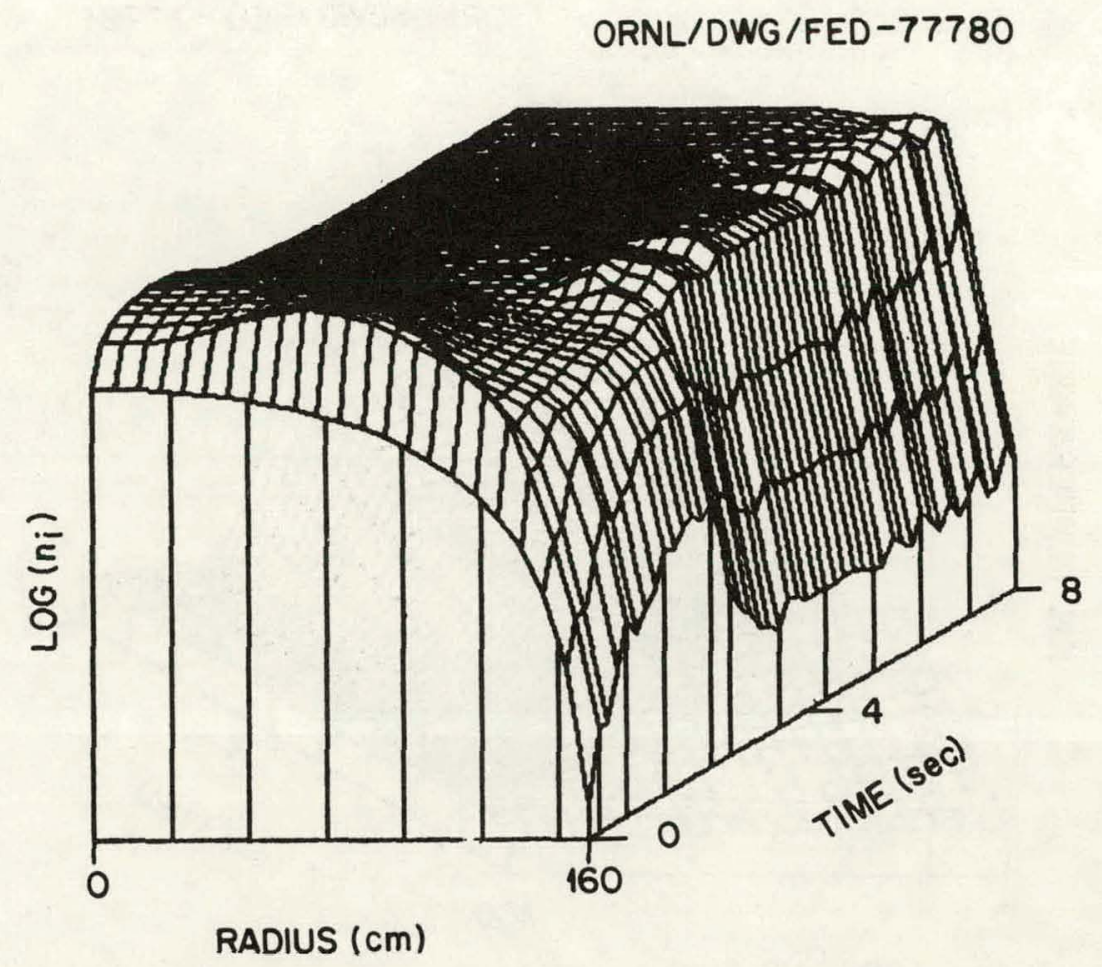

Fig. 18. Evolution of the density profile over $8 \mathrm{sec}$ for a small pellet, high plasma density case. A 2-mm-radius pellet is injected at $10^{3} \mathrm{~m} / \mathrm{sec}$ whenever the plasma density drops below $\bar{n}-10^{14}$ $\mathrm{cm}^{-3}$. This results in 177 pellets injected during the $8 \mathrm{sec}$ of simulation. Relatively small fluctuations in density are noted due to the relatively small pellet size. The density profile resembles the previous results with a low penetrating, continuous particle source. 


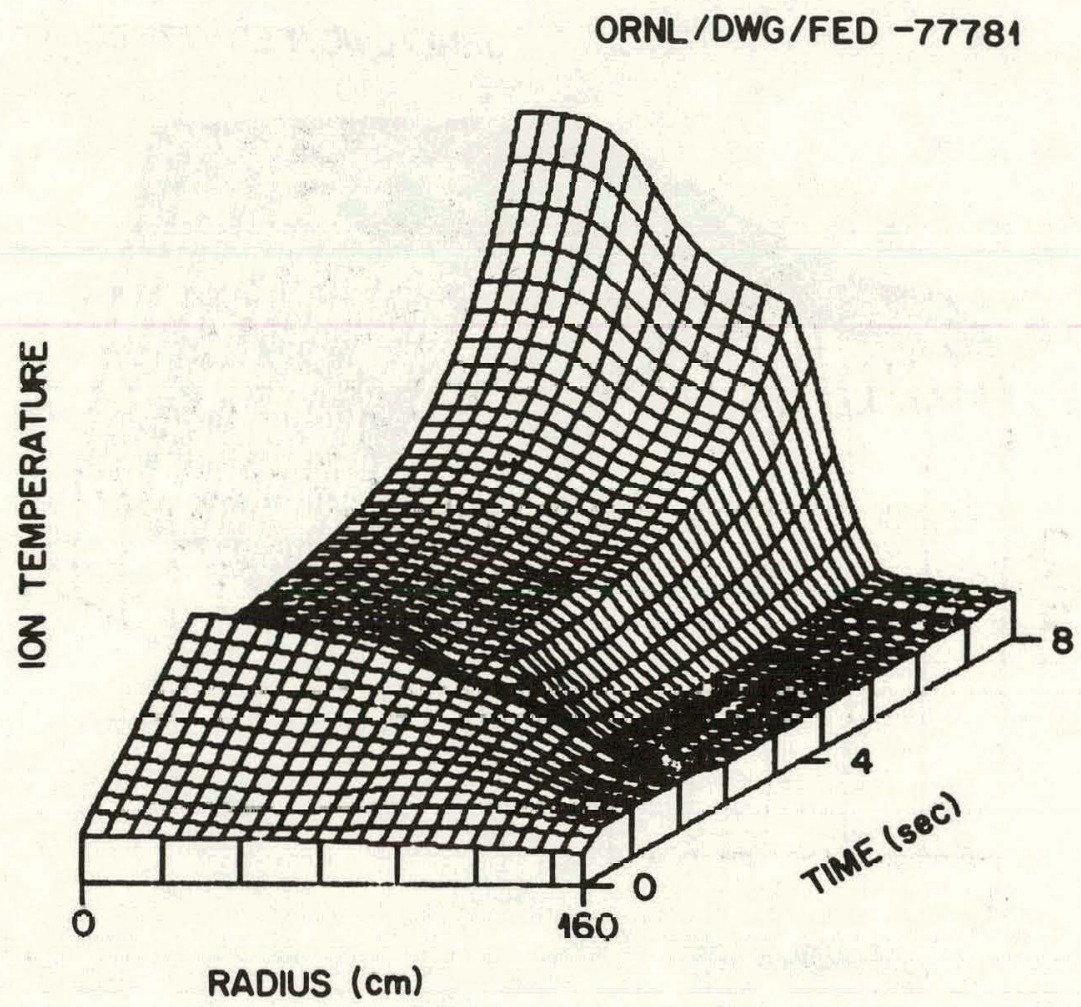

Fig. 19. Evolution of the ion temperature profile for the same case shown in Fig. 18. The hot ignited central core region (sharply detined because of local alpha heating) and the cold edge region resemble the low penetrating, continuous particle source results. 
plasma is well ignited and the temperature is rising rapidly. At $8 \mathrm{sec}$, the following values are seen: average ion temperature $=13.6 \mathrm{keV}$, average electron temperature $=15.0 \mathrm{keV}$, peak ion temperature $=35.1 \mathrm{keV}$, peak electron temperature $=35.4 \mathrm{keV}$, average density $=1.03 \times 10^{14} \mathrm{~cm}^{-3}$, peak density $=1.27 \times 10^{14} \mathrm{~cm}^{-3}$, average $\beta=6.4 \%$, and peak $\beta=19.4 \%$. Similar behavior is seen at lower densities with the obvious modifications which show deeper beam penetration and somewhat deeper pellet penetration.

These reactor-like conditions were reached in spite of the inclusion of the full trapped particle mode diffusion as described in WASH-1295.1 A zero-dimensional code would not have predicted that this system would ignite. 28 The one-dimensional treatment allows us to see the flattening of the density profile which occurs with shallowly penetrating pellets. The reduction of the density gradient diminishes the effect of the trapped particle mode diffusion terms as observed in Sect. 5. At $8 \mathrm{sec}$ the energy confinement time calculated from conduction, convection, and radiation losses is $5.3 \mathrm{sec}$ while that due to conduction and convection only is $9.5 \mathrm{sec}$. The particle confinement time is $0.44 \mathrm{sec}$, which reflects the fact that the particles are deposited very near the plasma edge. All of these times are much greater than zero-dimensional estimates of confinement times with trapped particle losses.

Figure 20 shows normalized values of the density-averaged ion temperature and alpha power as a function of time for a typical case. The irregularity of the temperature curve is due to fluctuations in the edge region from pellet injection. Note that the alpha power curve is relatively smooth, since the alpha power is strongly centrally peaked where the temperature and density are not fluctuating. A continuation of the curves shown in Fig. 20 is shown in Fig. 21 with an expanded time scale. The regular reductions in the average energy per plasma particle are due to pellet injection while the total fusion rate and thus the total power production continue to rise smoothly.

Figure 22 shows the evolution of the $\beta$ (pressure) profile for the same interval shown in Fig. 21. Since $\beta$ is proportional to the densitytemperature product (i.e., the stored energy density), and since a pellet carries very little stored energy, the $\beta$ fluctuations are quite small. 
ORNL /DWG /FED -77782

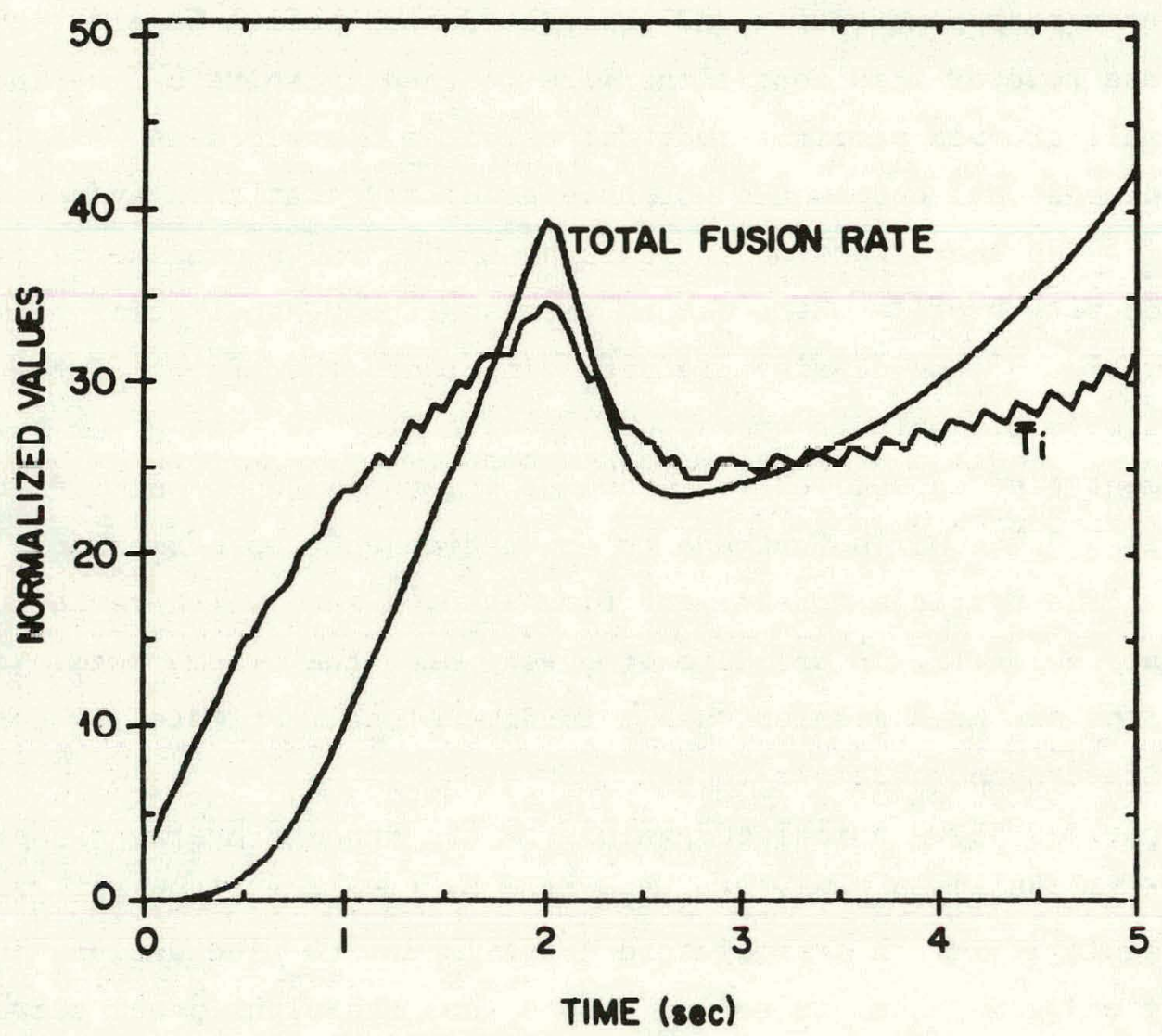

Fig. 20. The normalized values of the average ion temperature and total fusion rate as a function of time. The fluctuations in the ion temperature are due to fluctuations near the plasma edge due to pellets. The fusion rate is very strongly central peaked and basically unaffected by the edge fluctuations. 
ORNL/DWG/FED -77783

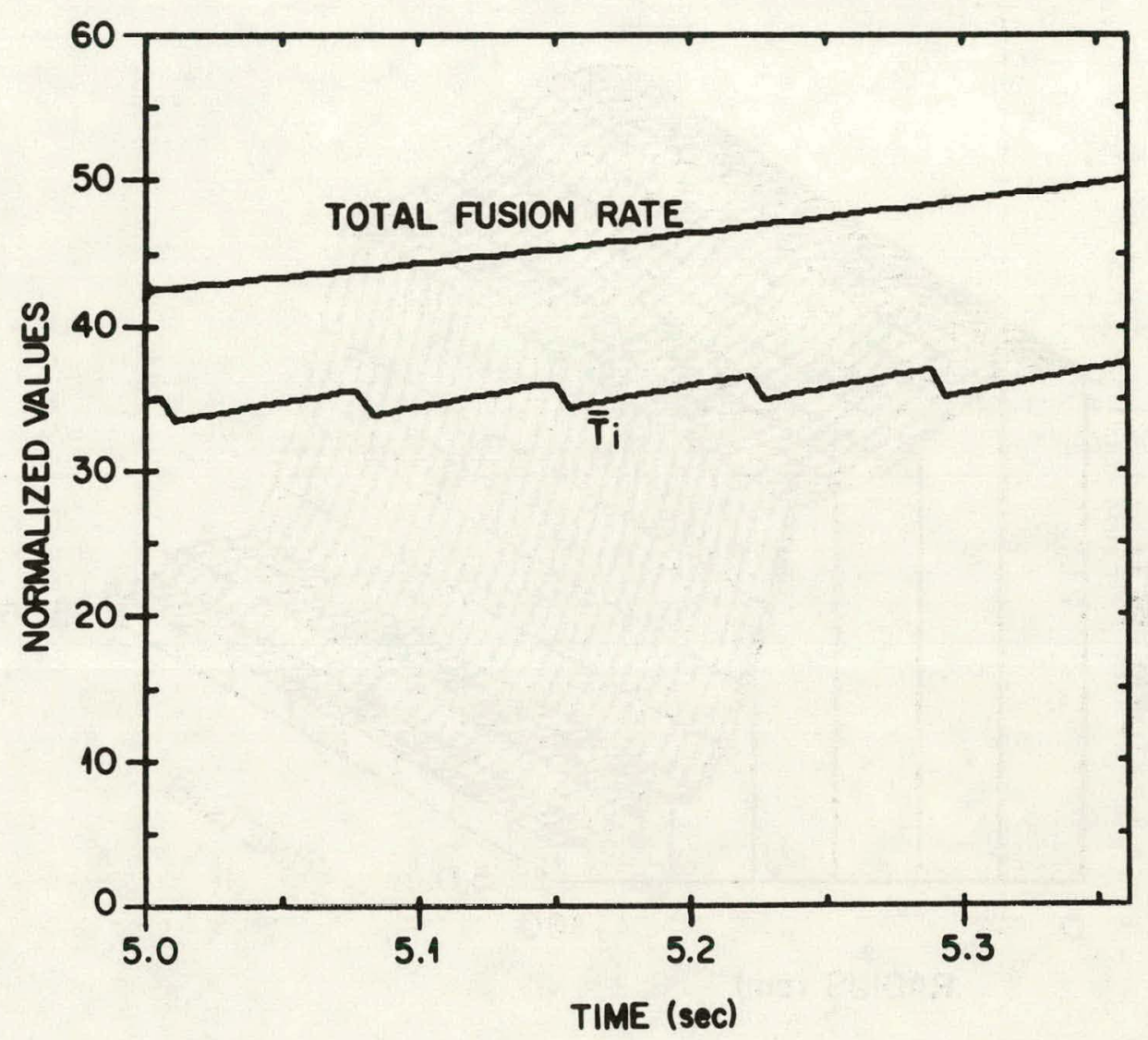

Fig. 21. A continuation of the plots illustrated in Fig. 20 using a finer time scale. The average ion temperature drops each time a pellet is injected at regular intervals. The total fusion rate rises continuously. 


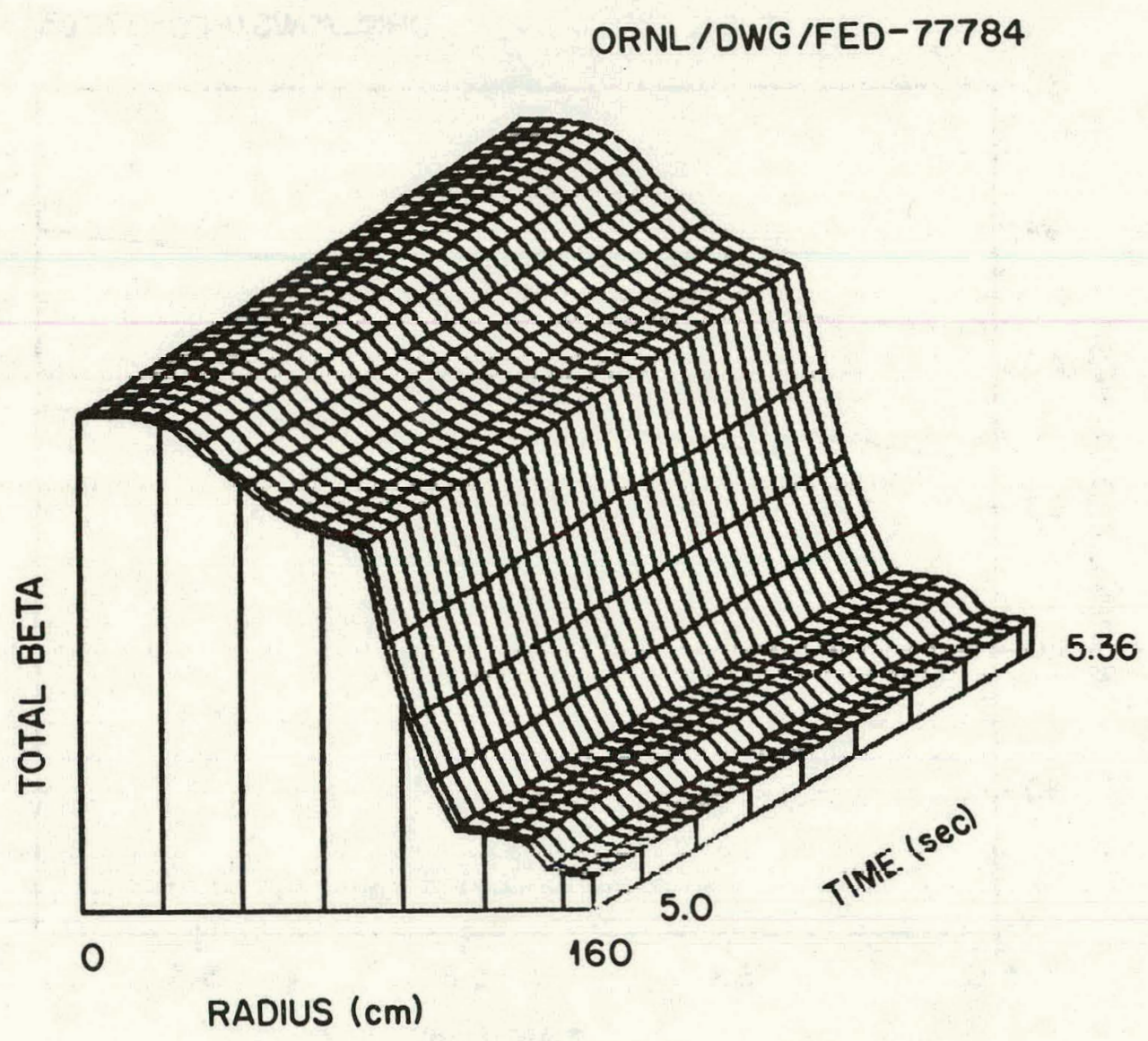

F'ig. '2'. Evolution of the total $\beta$ protile during the same time segment shown in Fig. 21. The density rises and the temperature drops after injection of a pellet. This occurs on a faster time scale than fluctuations in energy density and pressure. Since $\beta(r)$ is reasonably time independent, ideal MHD effects should be minimal. 
Figure 23 illustrates the effect of plasma density on the power required to ignite the plasma. Fueling is with 2-mm-radius pellets at a velocity of $10^{3} \mathrm{~m} / \mathrm{sec}$ in all cases. The ordinate is the minimum beam power required to ignite a TNS plasma in $2 \mathrm{sec}$ with a $200-\mathrm{keV}$ deuterium beam. The general trend is that higher density requires higher beam power to maintain a similar power per particle. However, at very high densities the beam is unable to penetrate, and the curves approach a limiting density asymptotically. At low densities, if the trapped particle modes.are not too severe, the ohmic heating reduces the required beam power. However, if the trapped particle losses are severe, then there is a minimum density which will not ignite at any temperature for the pellet parameters used here.

The most important point is that the spread in the data, which includes cases run with negligible trapped particle contributions (mainly neoclassical) and cases run with WASH-1295 full trapped particle contributions, is only about a factor of two in beam power. This is a clear indication that the trapped particle mode diffusion coefficients given in WASH-1295' can be very nearly "turned off" by shallowly penetrating pellets. The sharp density gradients are limited to the relatively high density, cold edge region in which the collisionality is high enough to reduce the potential for trapped particle microinstabilities.

To recapitulate, we have simulated discrete pellet injection into a TNS plasma. The temperature and density profiles are seen to fluctuate near the edge, but the $\beta$ and alpha power profiles do not fluctuate appreciably. The density profile is flattened as the pellets ablate near the edge. This tends to turn off the trapped particle mode diffusion, 29 allowing ignition through use of more optimistic parameters than those predicted by zero-dimensional treatments.

\section{SUMMARY}

Initial investigations of the effects of particle fueling profiles on plasma transport and plasma behavior have been discussed in this paper. In general, a controllable particle source profile may provide a significant amount of control over both equilibrium and dynamic behavior. Conduction and convection losses can be significantly reduced from global 


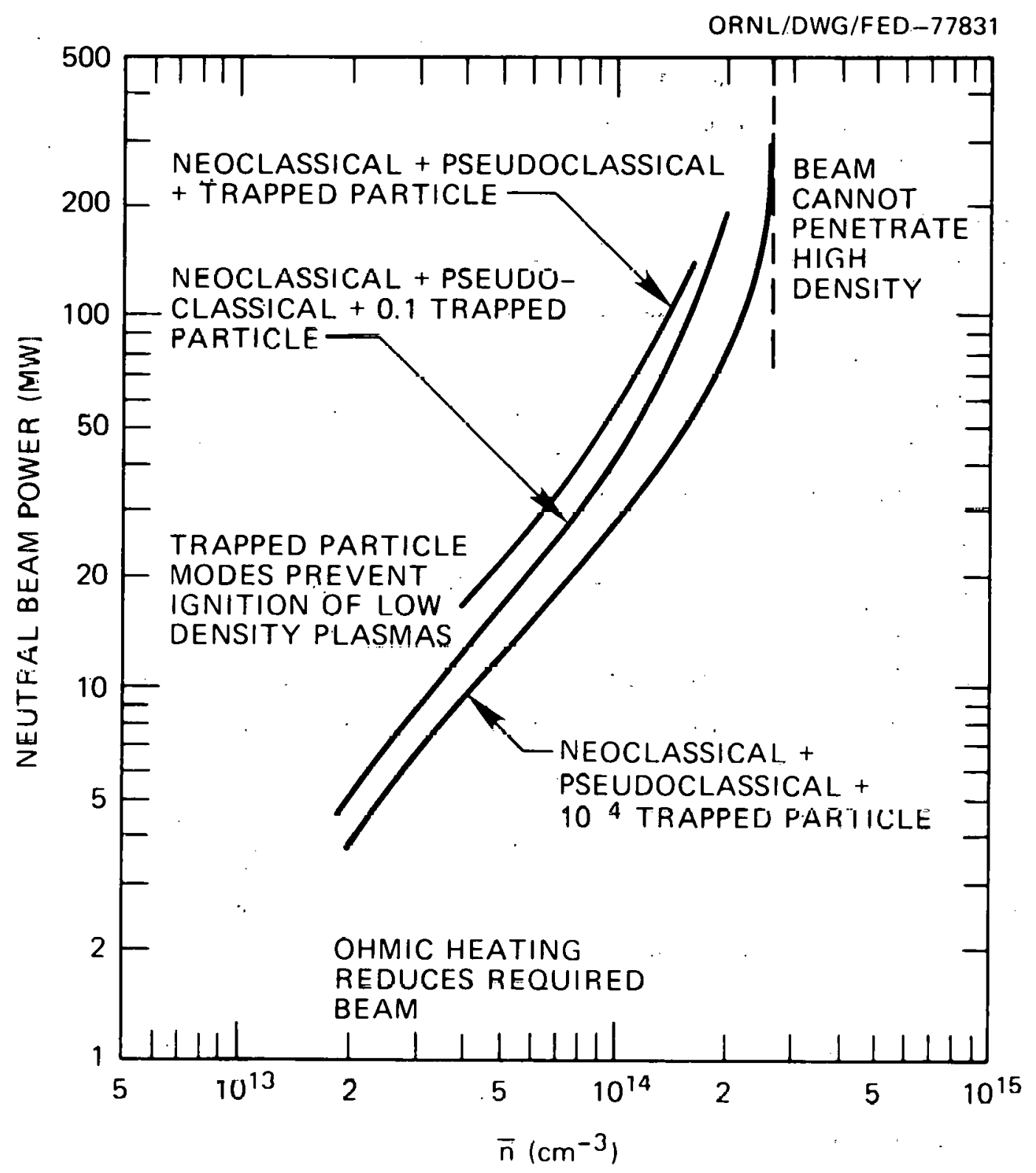

Fig. 23. The minimum beam power for ignition (200-keV beams on for $2 \mathrm{sec}$ ) as a function of plasma density. Pellets of $2-\mathrm{mm}$ radius injected at $10^{3} \mathrm{~m} / \mathrm{sec}$ are used for all cases. Note that there is only a factor of $\sim 2$ in required beam power between nevclassical and the full trapped particle model (WASH-1295). 
models for transport due to trapped particle microinstabilities because of approximations used for gradients.

The equilibrium calculations indicate that power output can be varied for a given density by varying the fueling profile. Lower density, lower power regimes, previously thought to be inaccessible with the trapped particle transport model used, can be reached with shallower fueling profiles.

A control1able particle source may be used to add external control over the plasma behavior during heating to ignition. Very low. penetration is easy to accomplish (e.g., gas puffing) but may increase losses near the plasma edge with the operation of a divertor. Very deep penetration to: the plasma center would be very difficult and may be undesirable if density gradient-driven trapped particle modes dominate the transport. Intermediate penetration depths provide an optimum in the heating-to-ignition power requirements for the model studied here.

The discrete pellet fueling model provides very encouraging results. Once the requirement that a pellet must penetrate to the center of the discharge is relaxed, estimates of pellet velocity can be significantly reduced. Fairly large fluctuations can occur in the density and temperature near the plasma edge with large pellets, but pressure ( $\beta$ ) fluctuations are very small. This should minimize ideal MHD effects. The central density and temperature do not fluctuate with low penetrating pellets, so power production in a reacting plasma should be continuous rather than oscillatory. No significant increase in beam attenuation is observed even in large ablation spikes in the edge density. $\Lambda 11$ of the above considerations allow relatively large ( $\leqslant 20-50 \%$ of the plasma content), low velocity pellets to be used.

Much further theoretical and experimental work needs to be done to adequately assess the effects of varying fueling profiles. The transport model certainly needs improvement. The ordering of scale lengths in determining the growth rates of the trapped particle modes under the conditions of edge fueling should be $|\nabla \mathrm{T}| \gg|\nabla \mathrm{n}|$. For any anomalous transport model the existence of "off-diagonal" terms in the transport matrix can be important. 
Experimental verification of the pellet ablation model is needed for higher density, hotter plasmas. Pellet injection experiments may also provide added insight in experimental determination of the transport coefficients. Theoretical and experimental analyses of limits on the pellet size must be performed. The kinetic and resistive MHD effects of a large, cold plasma source localized in both space and time require more thorough study.

Finite orbit effects and diffusion of fast alphas must be assessed to determine how much the alpha heating profile hroadens relative to the production profile. All of the above models should be incorporated in various startup scenarios (including small radius, low density concepts) and coupled to MHD equilibrium calculations to provide a more detailed analysis.

\section{APPENDIX A}

\section{BEHAVIOR OF THE PARTICLE BALANCE IN TOKAMAKS} UNDER THE CONDITIONS OF EDGE FUELING

Consider the one-dimensional form for the fluid particle balance for cylindrical geometry:

$\frac{\partial n}{\partial t}=-\frac{1}{r} \frac{\partial}{\partial r}(r \Gamma)+S_{p}(r)$,

where $S_{p}(r)$ represents the particle source from ionization of neutrals (beams, pellets, gas, etc.). The steady-state solution to Eq. (A.1) will be analyzed to give the direction in which the density profile tends to evolve. The steady-state solution is given by:

$\frac{1}{r} \frac{d}{d r}(r \Gamma)=s_{p}(r)$

Integrate Eq. (A.2) from $\circ$ to $r$ and use the boundary condition $\Gamma(0)=0$ to obtain the radial dependence of the particle flux in steady state. 
At the equilibrium (if one exists) the radial dependence of the particle source solely determines the radial flux:

$\left.\Gamma(r)\right|_{\text {equil. }}=\frac{1}{r} \int_{0}^{r} s_{p}\left(r^{\prime}\right) r^{\prime} d r^{\prime}$

The right-hand side of Eq. (A.3) is positive definite, and monotonically increasing provided the net particle source is nonnegative at all radii. Negative sources (sinks) can occur from recombination, which should be negligible in the hydrogenic particle balance but may play a role in the electron particle balance when impurities are present. Fusion of D-T will represent a small sink in the hydrogenic particle balance of ignited machines:

In order for Eq. (A.1) to behave like a diffusion equation, a dominant contribution to the particle flux must arise from the density gradient. In general, the particle flux may be written in the form:

$\Gamma=\Gamma_{n}+\Gamma_{T}+\Gamma_{p}$

where

$$
\begin{aligned}
& \Gamma_{\mathrm{n}} \equiv-\mathrm{D}_{\mathrm{n}} \frac{\partial \mathrm{n}}{\partial \mathrm{r}}, \quad \nabla \mathrm{n} \text {-driven particle flux } \\
& \Gamma_{\mathrm{T}} \equiv-\frac{\mathrm{nD}_{\mathrm{T}}}{\mathrm{T}} \frac{\partial \mathrm{T}}{\partial \mathrm{r}}, \nabla \mathrm{T} \text {-driven particle flux, Nernst effect } \\
& \Gamma_{\mathrm{p}} \equiv \text { pinch or expansion particle flux. }
\end{aligned}
$$

The "pinch" term $\Gamma_{p}$ is assumed to have no dependence on the density or temperature gradients but may be driven by the applied electric field as in the neoclassical model for transport. It is a pinch term if $\Gamma_{p}<0$ and an expansion term if $\Gamma_{p}>0$. The temperature gradient-driven flux (Nernst effect) can also act as a pinch term if $D_{T}<0$ for a centrally peaked temperature profile, in which case $\Gamma_{\mathrm{T}}<0$.

The density profile is really determined by a balance between the $\Gamma_{n}$ term, the pinch and Nernst terms, and the total particle source. The $\Gamma_{n}$ 
term must be nonzero in order for the density equation to be second order in the radial derivative of $n$, thereby making the equation parabolic. A second boundary condition on the density can then be applied. At equilibrium,

$\Gamma_{\mathrm{n}}=\Gamma-\Gamma_{\mathrm{T}}-\Gamma_{\mathrm{P}}$

$\frac{\partial \mathrm{n}}{\partial \mathrm{r}}=-\frac{1}{\mathrm{D}_{\mathrm{n}}}\left[\frac{1}{\mathrm{r}} \int_{0}^{\mathrm{r}} \mathrm{S}_{\mathrm{p}}\left(\mathrm{r}^{\prime}\right) \mathrm{r}^{\prime} \mathrm{d} \mathrm{r}^{\prime}-\Gamma_{\mathrm{p}}+\frac{\mathrm{n}}{\mathrm{T}} \mathrm{D}_{\mathrm{T}} \frac{\partial \mathrm{T}}{\partial \mathrm{r}}\right]$.

Several cases of Eq. (A.5) will now be considered.

Case A: $D_{T}=\Gamma_{p}=0, D_{n}>0$. At equilibrium $d n / d r \leqslant 0$ at all radii. This is the so-called "diagonal" term for the particle flux. When fueling is done only at the plasma edge such that $\mathrm{S}_{\mathrm{p}}=0$ in the central region, the density profile is flat in the central region $(d n / d r=0)$ in steady state. This is true regardless of the magnitude of $D_{n}$. The magnitude of $\nu_{n}$ determines how rapidly the steady state is reached. A small sink due to fusion of D-T $\left(S_{p}<0\right)$ may slightly hollow out the D-T density profile in an ignited plasma.

Case $B: \quad \Gamma_{p}=0, D_{n}>0, D_{T} \gtrless 0$. The addition of a temperature gradientdriven component of the flux will either help peak or hollow out the density profile. Again, consider the sourceless central region. The equilibrium density and temperature profiles in this region satisfy the equation

$\frac{d \ln (n)}{d \ln \left(I^{\prime}\right)}=-\frac{D_{T}}{D_{n}}$.

If $D_{T}$ and $D_{n}$ are both positive (fluxes down the gradients), the density and temperature will have opposite gradients. For centrally peaked heating, the temperature should be centrally peaked and the density profile hollowed out to obtain equilibrium. The relative shape of the profiles is determined 
by the ratio of $D_{T}$ to $D_{n}$, and the rate at which equilibrium is approached is determined by the magnitude of $D_{T}$ and $D_{n}$.

For the banana regime of neoclassical theory, $\mathrm{D}_{\mathrm{T}}<0$. Hydrogenic particles diffuse up the temperature gradient. This can lead to centrally peaked hydrogenic density profiles even when no fueling occurs at the center. At the same time, higher $Z$ particles diffuse down the temperature gradient in the banana regime. This may act as a mechanism for removing fusion-produced alphas in an ignited machine and for screening high $\mathrm{Z}$ materials from entering the plasma core.

Case C: $\Gamma_{p} \neq 0$. The pinch or expansion term, $\Gamma_{p}$, affects the density profile in much the same manner as the temperature gradient-driven component of the flux. If $\Gamma_{p}$ represents a pinch term (as for the Ware pinch of neoclassical theory), the density profile will tend to peak at the center even in the absence of central fueling. The density profile tends to be hollowed out when $\Gamma_{p}>0$.

What is needed from the kinetic theory of the dissipative trapped particle microinstabilities or other anomalous transport mechanisms is to determine whether there are any strong "off-diagonal" contributions to the particle flux. In the final analysis these terms may determine whether or not edge fueling is feasible. A more difficult task is to determine whether there are any mass effects in the anomalous diffusion of deuterium and tritium which preferentially allow one species to diffuse inward more readily than the other.

\section{APPENDIX B}

\section{DATA FROM EQUILIBRIUM PROFILE STUDY}

In performing the analysis for Sect. 4, in which a search was made for thermally stable equilibria, the effects of multiplying the WASH-1295 1 transport coefficients by an arbitrary fraction $\alpha_{\mathrm{TP}}$ were investigated briefly. The consequences were obvious and were therefore not reported in the main body of this report. The essential features of varying $\alpha_{\mathrm{TP}}$ from 1 down to 0.1 can be seen from the data in Table B.1 and from Fig. B.I. As $\alpha_{\text {TP }}$ decreases, the confinement time increases; as a consequence, the minimum density which the plasma can have and still maintain an ignited 

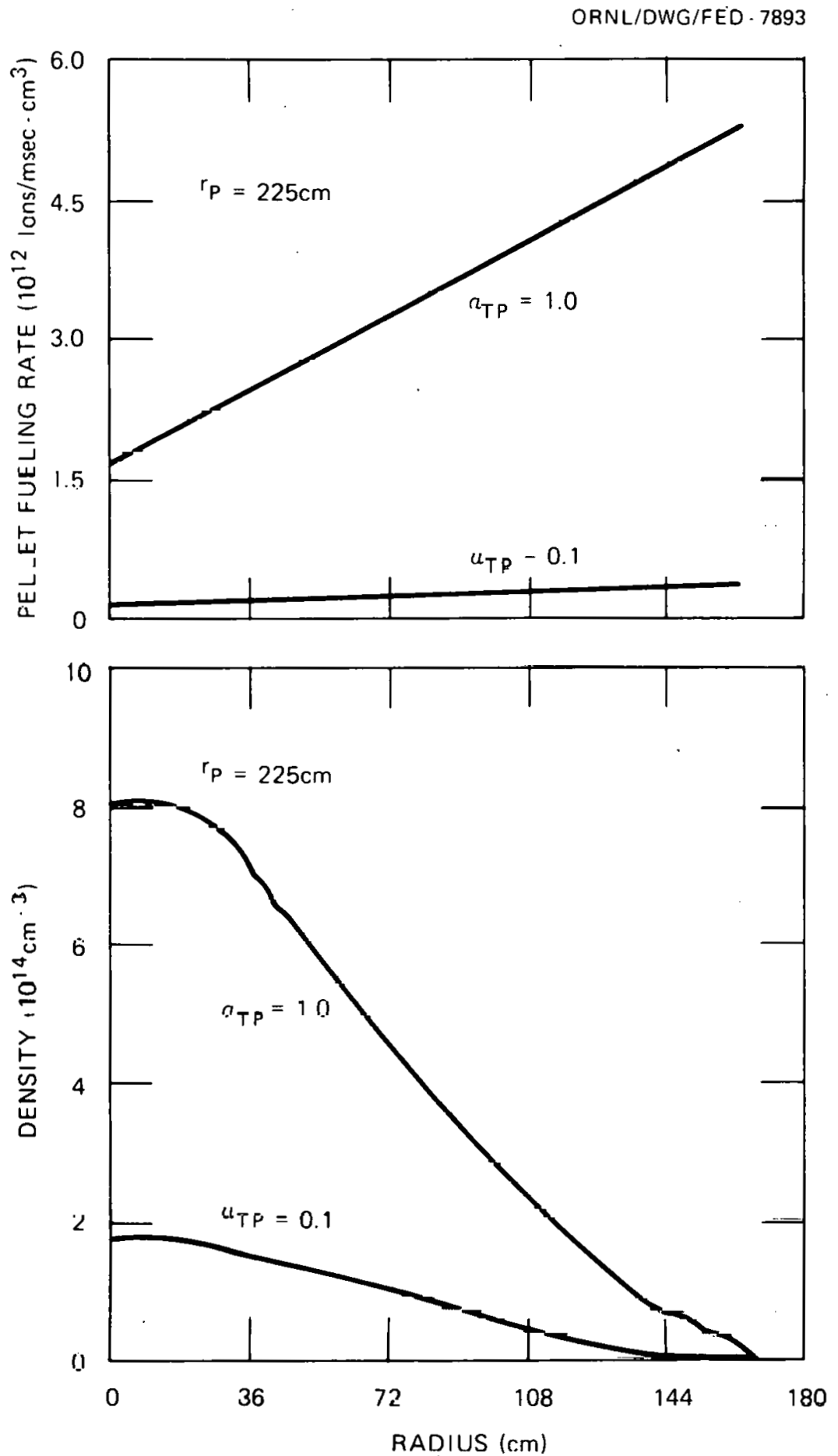

B.1. Reducing trapped particle diffusion coefficients by a factor of 10 reduced required fueling rate by a factor of 20 , in a typical case. 
equilibrium is reduced. Since $\tau_{p}$ increases, the particle loss rate decreases; therefore, the fueling rate can be decreased for the same penetration depth.

Table B.1 is included to demonstrate the trends in the data as well as the spread. It must be reiterated that the uncertainties in the transport coefficients are large; therefore, one should view the numerical values with caution and pay more attention to the trends.

The last colum in. Table B.I gives the ratio of the global energy confinement time to the actual particle confinement time. The particle confinement time, $\tau_{p}$, was computed from the known source rate required to keep the average density fixed; therefore, it is the true confinement time. It does not, in general, correspond to $a^{2} / 4 D$ where $D$ is the diffusion coefficient evaluated with some average plasma parameters [e.g.; $\overline{\mathrm{T}}_{i}, \overline{\mathrm{T}}_{e}$, $\overline{\mathrm{n}} / \mathrm{a}, \mathrm{B}_{\mathrm{p}}(\mathrm{a})$, etc. $]$, and $\mathrm{a}_{\mathrm{i}}$ is the plasma radius. As can be seen $\left(\tau_{E}\right)_{G l o b a l} / \tau_{p}>1$, which is characteristic of a plasma dominated by diffusion losses rather than by conduction, charge exchange, and radiation 1osses.

For a plasma with a divertor, if one uses the definitions for $\tau_{p}$ and $\left(\tau_{E}\right)_{G l o b a l}$ given below, a simple relationship can be derived for $\tau_{E G} / \tau_{p}$. Definition of $\tau_{\mathrm{p}}$ :

$\frac{\bar{n} V}{\tau_{p}} \equiv \underset{\text { separatrix }}{\phi} \cdot \dot{d} \cdot \dot{A} \cdot s \cdot$

Definition of $\left(\tau_{E}\right)_{\text {Global }}$ :

$\frac{3 / 2^{\bar{n}\left(\bar{T}_{e}+\bar{T}_{i}\right) V}}{\tau_{E G}} \equiv P_{\text {Loss }}=P_{\text {rad }}+P_{C X}+P_{\text {CONDUCTION }}+P_{\text {CONVECTION }}$, (B.2)

where $\mathrm{V}=$ plasma volume out to separatrix and all barred quantities ( $)$ refer to volume averages over the plasma from $r=0$ to $r=r_{s}$, the separatrix radius. All loss terms are integrated over the plasma out to the separatrix; thus $\mathrm{P}_{\mathrm{CX}}$, for example, does not include charge-exchange losses in the divertor zone. 
Looking at the last term in Eq. (B.2), we note

$$
\begin{aligned}
P_{\mathrm{CONV}} \equiv & \int \nabla \cdot\left[\frac{3}{2}\left(\mathrm{~T}_{e}+\mathrm{T}_{1}\right) \vec{\Gamma}\right] \mathrm{dV} \\
= & \oint \frac{3}{2}\left(\mathrm{~T}_{\mathrm{e}}+\mathrm{T}_{1}\right) \vec{\Gamma} \cdot \overrightarrow{\mathrm{dA}} \\
& \text { separatrix } \\
= & \frac{3}{2}\left(\mathrm{~T}_{\text {es }}+\mathrm{T}_{1 \mathrm{~s}}\right) \Gamma_{s} \mathrm{~A}_{s},
\end{aligned}
$$

where the s subscript refers to the quantity being evaluated at the ocparatris.

Combining Eqs. (B.1), (B.2), and (B.3), it is simple to show that

$\frac{\tau_{E G}}{\tau_{p}}=\frac{\overline{\mathrm{T}}_{e}+\overline{\mathrm{T}}_{i}}{\mathrm{~T}_{\text {es }}+\mathrm{T}_{\text {is }}}\left(1-\frac{\tau_{E G}}{\tau_{\Delta}}\right)$,

where $\tau_{\Delta}$ is the global energy confinement time for the plasma not including convection losses and

$$
\frac{\tau_{E G}}{\tau_{\Delta}} \equiv \frac{P_{\text {rad }}+P_{C X}+P_{C O N D}}{P_{\text {rad }}+\bar{P}_{C X}+\vec{P}_{C O N D}+\bar{P}_{\text {CONN }}}
$$

= fraction of energy lost from plasma by radiation, $\mathrm{CX}$, and conduction to the plasma in the divertor zone.

Usually for $z_{\text {eff }} \cong 1$ plasmas we find $\left(\bar{T}_{e}+\bar{T}_{i}\right) /\left(T_{e s}+T_{i s}\right) \approx 10$, which implies that $\tau_{E G} / \tau_{p}=10\left(1-\tau_{E G} / \tau_{\Delta}\right)$. Therefore the fraction of energy lost (across the separatrix) due to thermal conduction, charge exchange, and radiation must be greater than $90 \%$ in order for $\tau_{\text {Ff }}$ to be legs than " $\mathrm{p}$ " 


\section{Definitions for use with Table B.I}

$r_{p}=$ profile penetration depth $(\mathrm{cm})$ used for source profile $s_{p}(r)=s_{p}(a)\left[1-(a-r) / r_{p}\right], r>\left(a-r_{p}\right)$

$S_{0}=$ total injected Ion source rate $\left(\mathrm{msec}^{-1}\right)$, i.e., fueling rate $\overline{\mathrm{n}}=$ average ion density $\left(\mathrm{cm}^{-3}\right)$

$\mathrm{n}_{0}=$ Ion density at center of plasma $\left(\mathrm{cm}^{-3}\right)$

$P_{\alpha}=$ total alpha power produced in plasma (MW)

$\overline{\mathrm{T}}_{i}=$ density-averaged ion energy (keV)

$T_{i}(0)=$ ion temperature at center of plasma (keV)

$\tau_{p} \equiv$ particle confinement time (msec), calculated from $\bar{n}_{i} V / S_{0}$

$\tau_{E} \equiv$ Global energy confinement time (msec), calculated from $3 \overline{\mathrm{n}}_{\mathrm{i}} \overline{\mathrm{T}}_{\mathrm{i}} / \mathrm{p}_{\alpha}$

$\alpha_{\mathrm{TP}}=$ multiplier for trapped particle mode transport coefficients $\left(\alpha_{\mathrm{TP}}=0.1\right.$ implies 0.1 of WASH-1295 values used for $D, X_{e}$, and $\left.x_{i}\right)$

$\frac{\tau_{E}}{\tau_{P}}=4.8 \times 10^{-19} \overline{\mathrm{T}}_{i}(\mathrm{keV}) \mathrm{s}_{0}\left(\mathrm{msec}^{-1}\right) / \mathrm{p}_{\alpha}(\mathrm{MW})$ 
Table B.1. Data for equilibrium cases

\begin{tabular}{|c|c|c|c|c|c|c|c|c|c|c|}
\hline \multirow{2}{*}{ Run $\#$} & \multirow{2}{*}{$r_{p}(c m)$} & \multirow{2}{*}{ H/msec } & $\overline{\mathbf{n}}$ & $n(0)$ & \multirow{2}{*}{$P \alpha(M W)$} & \multirow{2}{*}{$\overline{\mathrm{T}}_{i} \mathrm{keV}$} & \multirow{2}{*}{$\mathrm{T}_{1}(0) \mathrm{keV}$} & \multirow{2}{*}{$\tau_{p}($ msec $)$} & \multirow{2}{*}{$\alpha_{\mathrm{TP}}$} & \multirow{2}{*}{$\tau_{E} / \tau_{P}$} \\
\hline & & & $10^{14} \mathrm{~cm}^{-3}$ & $10^{14} \mathrm{~cm}^{-3}$ & & & & & & \\
\hline 2249 & 115 & $2.2 \times 10^{20}$ & 1.9 & 3.4 & 295 & 11.6 & 71 & 213 & 1.0 & 4.2 \\
\hline 655 & 115 & 1.57 & 1.7 & 3.1 & 218 & 10.8 & 72 & 273 & 1.0 & 3.7 \\
\hline 814 & 115 & 0.81 & 1.15 & 2.2 & 91 & 8.7 & 52 & 355 & 1.0 & 3.7 \\
\hline 749 & 115 & 1.35 & 1.53 & 2.7 & 173 & 10.6 & 70 & 283 & 1.0 & 4.0 \\
\hline 820 & 115 & 1.17 & 1.34 & 2.4 & 121 & 9.4 & 61 & 286 & 1.0 & 4.4 \\
\hline 847 & 150 & 9.7 & 2.75 & 6.5 & 710 & 9.73 & 25.7 & 71 & 1.0 & 6.4 \\
\hline 523 & 125 & 5.0 & 2.13 & 4.2 & 361 & 9.5 & 38.0 & 107 & 1.0 & 6.3 \\
\hline 532 & 125 & 7.5 & 2.76 & 5.5 & 793 & 12.0 & 52.7 & 92 & 1.0 & 5.5 \\
\hline 1028 & 125 & 6.3 & 2.34 & 4.6 & 5.18 & 10.8 & 45.6 & 93 & 1,0 & 6.3 \\
\hline$\overline{700}$ & 175 & 10.0 & 2.75 & 7.3 & 672 & 9.2 & 21.6 & 69 & 1.0 & 6.6 \\
\hline 751 & 225 & 10.4 & 2.74 & 8.1 & 640 & 8.8 & 18.6 & 66 & 1.0 & 6.9 \\
\hline 927 & flat $10^{6}$ & 11.0 & 2,46 & 9.1 & 444 & $\% .9$ & 14.5 & 56 & 1.0 & 9.4 \\
\hline 452 & 225 & 8.91 & 2.49 & 7.4 & 543 & 8.9 & 19.4 & 70 & 1.0 & 7.0 \\
\hline 626 & 225 & 8.77 & 2.50 & 7.4 & 497 & 8.6 & 17.9 & 71 & $n .9$ & 7.3 \\
\hline 441 & 225 & 8.83 & 2.48 & 7.4 & 616 & 9.4 & 21.0 & 70 & 0.8 & 6.5 \\
\hline 710 & 225 & $8: 95$ & 2.48 & 7.11 & 792 & 10.8 & 25.1 & $6 p$ & 0.6 & 5.9 \\
\hline 804 & 225 & 7.76 & 2.30 & 6.8 & 621 & 16.2 & 23.6 & 74 & 0.6 & 6.1 \\
\hline 931 & 225 & 6.68 & 2.11 & 6.3 & 477 & 9.71 & 22.0 & 79 & 0.6 & 6.5 \\
\hline 1036 & 225 & 5.67 & 1.92 & 5.7 & 356 & 9.2 & 20.5 & 85 & 0.6 & 7.0 \\
\hline 603 & 225 & 4.70 & 1.73 & 5.2 & 257 & 8.7 & 19.0 & 92 & 0.6 & 7.6 \\
\hline 701 & 225 & 3.88 & 1.54 & 4.6 & 178 & $8 . \dot{2}$ & 17.6 & yy & 0.6 & 8.6 \\
\hline 1040 & 225 & $3.85 \times 10^{20}$ & 1.54 & 4.75 & 226 & 8.96 & 21.3 & 100 & 0.5 & 7.3 \\
\hline 445 & 225 & 3.88 & 1.54 & 4.74 & 271 & 9.84 & 24.2 & 100 & 0.4 & 6.8 \\
\hline 745 & 225 & 3.96 & 1.54 & 4.72 & 338 & 11.2 & 28.8 & 97 & 0.3 & 6.3 \\
\hline 910 & 225 & 3.58 & 1.35 & 1.06 & 292 & 12.3 & 41.9 & 95 & 0.3 & 7.2 \\
\hline 444 & 225 & 2.39 & 1.16 & 3.68 & 149 & 9.5 & 25.6 & 121 & 0.3 & 7.3 \\
\hline 606 & 225 & 1.63 & 0.37 & 3.07 & 92 & ח.ก.s & 27.1 & $14 A$ & 0.7 & 7.5 \\
\hline 646 & 225 & 1.05 & 0.78 & 2.50 & 49 & 7.98 & 20.2 & 185 & 0.3 & 8.2 \\
\hline 734 & 225 & 0.62 & 0.59 & 1.89 & 21 & 7.02 & 17.1 & 238 & 0.3 & 10.0 \\
\hline 450 & 225 & 0.61 & 0.59 & 1.87 & 32 & 8.42 & 22.1 & 241 & 0.2 & 7.7 \\
\hline 531 & 225 & 0.57 & 0.60 & 1.86 & 55 & 11.6 & 36.0 & 263 & 0.1 & 5.8 \\
\hline 645 & 200 & 0.61 & 0.60 & 1.79 & 55 & 11.8 & 39.6 & 246 & 0.1 & 6.3 \\
\hline 639 & 200 & 0.44 & 0.55 & 1.57 & 45 & 11.8 & 38.5 & 313 & 0.1 & 5.5 \\
\hline 745 & 200 & 0.39 & 0.50 & 1.45 & 32 & 10.5 & 33.0 & 320 & 0.1 & 6.1 \\
\hline
\end{tabular}


ACKNOWLEDGMENTS

The authors would like to acknowledge helpful conversations with J. F. Clarke, R. J. Colchin, and C. A. Foster. 


\section{REFERENCES}

1. S. 0. Dean et al., "Status and Objectives of Tokamak Systems for Fusion Research," WASH-1295, USAEC, Washington, D.C. (1974).

2. F. L. Hinton and T. B. Moore, Nucl. Fusion 14, 639 (1974).

3. S. L. Milora and C. A. Foster, "ORNL Neutral Gas Shielding Model for Pellet-Plasma Interactions," ORNL/TM-5776, Oak Ridge National Laboratory, Oak Ridge, Tennessee (May 1977).

4. S. I. Braginskii, "Reviews of Plasma Physics," Ist ed., Vol. 1. Consultants Bureau, New York, 1965.

5. R. D. Hazeltine, F. L. Hinton, and M. N. Rosenbluth, Phys. Fluids 16, 1645 (1973).

6. A. T. Mense, Ph.D. Thesis, Department of Nuclear Engineering, University of Wisconsin, Madison, Wisconsin (1977).

7. R. W. Conn and J. Kesner, J. Nuc1. Mater. 63, 1 (1976).

8. J. A. Rome, Y-K. M. Peng, and J. A. Holmes, "Injection Heating Scenarios for TNS," ORNL/TM-5931, Oak Ridge National Laboratory, Oak Ridge, Tennessee (July 1977).

9. L. A. Berry et al., "Confinement Neutral Beam Injection Studies on ORMAK," Proc. 6th IAEA Conf. on Plasma Physics and Controlled Nuclear Fusion Research, Berchtesgaden, West Germany (October 6-13, 1976).

10. S. T. Butler and M. J. Buckingham, Phys. Rev. 126, 1 (1962).

11. M. Dawson, H. P. Furth, and F. H. Tenney, Phys. Rev. Lett. 26, 1156 (1971).

12. J. G. Cordey, W. C. F. Core, and J. Sheffield, Nucl. Fusion 15, 755 (1975).

13. D. G. McAlees, Ph.D. Thesis, Department of Nuclear Engineering, University of Wisconsin, Madison, Wisconsin (1974).

11. W. A. Huulberg, Ph.D. Thec1e, Department of Nuclcar Enginecring, University of Wisconsin, Madison, Wisconsin (1977).

15. D. J. Sigmar and H. C. Chan, "Anomalous Alpha Particle Transport in Fusion Plasma," to be published as ORNL/TM-6027, Oak Ridge National Laboratory, Oak Ridge, Tennessee. 
16. W. G. Manheimer, "An Introduction to Trapped-Particle Instability in Tokamaks," TID-27157, Technical Information Center, Department of Energy, Washington, D.C. (1977).

17. W. A. Houlberg and R. W. Conn, Nucl. Sci. Eng. 64, 141 (1977).

18. P. B. Parks, R. J. Turnbu11, and C. A. Foster, Nuc1. Fusion 15, 557 (1977).

19. D. F. Vaslow, "Scaling Law for the Ablation of a Hydrogen Pellet in a Plasma," IEEE Transactions on Plasma Science, Vol. P5-5, No. 1 (March 1977).

20. D. J. Rose, (Culham Laboratory Technology Division), memorandum No. $82,1968$.

21. S. L. Gralnick, Nucl. Fusion 13, 703 (1973).

22. C. T. Chang, Nucl. Fusion 15, 595 (1975).

23. C. A. Foster, R. J. Colchin, S. L. Milora, K. Kim, and R. J. Turnbull, "Solid Hydrogen Pellet Injection Into the ORMAK Tokamak," ORNL/TM-5897, Oak Ridge National Laboratory, Oak Ridge, Tennessee (June 1977), to be published in Nucl. Fusion.

24. W. T. Miles, R. Thompson, and A. E. S. Green, J. App1. Phys. 43, 678 (1972).

25. D. G. McAlees et a1., ORNL/TM-5573, Oak Ridge National Laboratory, Oak Ridge, Tennessee (October 1976). W. M. Stacey, Jr. et al., "Tokamak Experimental Power Reactor Conceptual Design," ANL/CTR/76-3, Argonne National Laboratory, Argonne, Illinois (1976). Fusion Study Group, GA-A14000, General Atomic Company, San Diego, California (1976).

26. R. W. Conn et al., "A High Performance Noncircular T'okamak Power Reactor Design Study-UWMAK-111," Proc. 6th IAEA Conf. on Plasma Physics and Controlled Nuclear Fusion Research, Berchtesgaden, West Germany (October 6-13, 1976).

27. J, Kesner (University of Wisconsin), private communication, 1976.

28. S. E. Attenberger, F. B. Marcus, and D. G. McAlees, "Dynamics of Tokamak Plasma Exper1ments and Reactors," ORNL/TM-5509, Oak Ridge National Laboratory, Oak Ridge, Tennessee (November 1976).

29. J. F. Clarke, "An Improved Est1mate of Trapped Ion Mode Energy Loss From Tokamak Reactors," ORNL/TM-5860, Oak Ridge National Laboratory, Oak Ridge, Tennessee (May 1977). 


\section{THIS PAGE}

\section{WAS INTENTIONALLY \\ LEFT BLANK}


ORNL/ TM-6026

Dist. Category UC-20a, d, and $\mathrm{g}$

\section{INTERNAL·DISTRIBUTION}

1. L. A. Berry

2. .T. D. Callen

3. R. A. Dand 1

4. R. A. Dory

5. G. G. Kelley

6. H. H. Haselton

7. P. N. Haubenreich

8. M. S. Lube11

9. 0. B. Morgan

10. H. Postma

11. M. W. Rosenthal
12. J. Sheffleld

13. D. Steiner

14-39. A. T. Mense

40-41. Laboratory Records Department

42. Laboratory Records, ORNL-RC

43. Document Reference Section

44-45. Central Research Library

46. Fusion Energy Division Library

47. Fusion Energy Division

Communications Center

48. ORNL Patent Office

\section{EXTERNAL DISTRIBUTION}

49. Bibliothek, Max-Planck Institute für Plasmaphysik, 8046 Garching bei München, Federal Republic of Germany

50. Bibliothèque, Service du Confinement des Plasmas, C.E.A., B.P. No. 6, 9.2, Fontenay-aux Roses (Seine), France

51. Lung Cheung, Department of Electronics, University Science Center, The Chinese University of Hong Kong, Shatin, N.T., Hong Kong

52. J. F. Clarke, Office of Fusion Energy, G-234, Department of Energy, Washington, DC 20545

53. D. Cohn, Massachusetts Institute of Technology, Cambridge; MA 02139

54. R. W. Conn, Fusion Technology. Program, Nuclear Engineering Department, University of Wisconsin, Madison, WI 53706

55. CTR Library, c/o Alan F. Haught, United Technologies Research Laboratory, East Hartford, CT 06108

56. CTR Reading Room, c/o Allan N. Kaufman, Physics Department, University of California, Berkeley, CA 94720

57. Hatice Cullingford, Office of Fusion Energy, G-234, Department of Energy, Washington, DC 20545

58. J. Narl Davidson, School of Nuclear Engineering, Georgia Institute of Technology, Atlanta, GA 30332

59. Documentation S.I.G.N., Départment de la Physique du. Plasma et de la Fusion Controlée, Association EURATOM-CEA sur la Fusion, Centre d'Études Nucléaires, B.P. 85, Centre du TRI, 38041 Grenoble, Cedex, France

60. W. R. E11is, Office of Fusion Energy, G-234, Department of Energy, Washington, DC 20545

61. G. A. Emmert, Nuclear Engineering Department, University of Wisconsin, Madison, WI 53706

62. Harold K. Forsen, Exxon Nuclear Co., Inc., 777 106th Avenue, N.E., C-000777, Bellevue, WA 98009 
63. Harold P. Furth, Princeton Plasma Physics Laboratory, Princeton University, Forrestal Campus, P.0. Box 451, Princeton, NJ 08540

64. Roy W. Gould, California Institute of Technology, Mail Stop 116-81, Pasadena, CA 91125

65. Charles R. Head, Office of Fusion Energy, G-234, Department of Energy, Washington, DC 20545

66. Robert L. Hirsch, Exxon Research and Engineering, P.0. Box 101, Florham Park, NJ 07932

67. Raymond A. Huse, Manager, Research and Development, Public Service Gas and Electric Company, 80 Park Place, Newark, NJ 07101

68. T. Hsu, Office of Fusion Energy, G-234, Department of Energy, Washington, DC 20545

69. V. K'. Ivanov, Physical-Technical Institute of the Ukranian Academy of Sciences, Sukhumi, U.S.S.R.

70. D. L. Jassby, Princeton Plasma Physics Laboratory, P.0. Box 451, Princeton, NJ 08540

71. A. Kadish, Office of Fusion Ellergy, G-234, Department of Energy, Washington, DC 20545

72. L. M. Kovrizhnikh, Lebedev Institute of Physics, Academy of Sciences of the U.S.S.R., Leninsky Prospect 53, Mnsrowt, U.S.S.R.

73. Guy Laval, Groupe de Physique Théorique, Ecol,e Polytechnique, 91. Palaiseau, Paris, France

74. Library, Centre de Recherches en Physique des Plasma, 21 Avenue des Bains, 1007, Lausanne; Switzerland

75: Library, Culham Laboratory, United Kingdom, Atomic Energy Authority, Abingdon, Oxon, OX14 3DB, United Kingdom

16. Library, FOM-Institut voor.Plasma - Fysica, Rijnhuizen, Jutphaas, Netherlands

77. Library, Institute for Plasma Physics, Nagoya University, Nagoya, Japan 464

78. Iibrary, Intcrnational Centre ful Theoretlcal Physic̄s, l'rieste, Italy

79. Library, Laboratorio Gas Ionizzati, Frascati, Italy

80. Dsumber G. Lominadze, Academy of Sciences of the Georgian S.S.R., 8 Dzerzhinski St., 38004, Tbilisi, U.S.S.R.

81. Oscar P. Manley, Office of Fusion Energy, त-234, Department of Energy, Washington, DC 20545

82. D. G. McAlees, Exxon Nuclear Co., Inc., Research and Technology Laser Enrichment Department, 2955 George Washington Way, Richland, WA 99352

83. T.E. McCune, School of Engineering, Department of Aeronaur1cs and Astronautics, Bldg. 37-391, Massachusetts Inst1tute of Technology, Cambridge, MA. 02139

84. Claude Mercier, Service du Theorie des Plasmas, Centre d'Études Nucléaires, Fontenay-aux-Roses (Seine), France

85. K. G. Moses, Office of Fusion Energy, G-234, Department of Energy, Washington, DC 20545

86. Michael Murphy, Office of Fusion Energy, G-234, Department of Energy, Washington, DC 20545 
87. D. Pfirsch, Institute for Plasma Physics, 8046 Garching bel Minchen, Federal Republic of Germany

88. Plasma Physics Group, Department of Engineering Physics, Australian National University, P.0. Box 4, Canberra A.C.T. 2600, Australia

89. Robert E. Price, Office of Fusion Energy, G-234, Department of Energy, Washington, DC 20545

90. A. Rogister, Institute for Plasma Physics, KFA, Postfach 1913, D-5170, Jülich 1, Federal Republic of Germany

91. W. Sadowski, Office of Fusion Energy, G-234, Department of Energy, Washington, DC 20545

92. V. D. Shafranov, I. V. Kurchatov Institute of Atomic Energy, 46 Ulitsa Kurchatova, P.0. Box 3402, Moscow, U.S.S.R.

93. Yu. S. Sigov, Institute of Applied Mathematics of the U.S.S.R. Academy of Sciences, Miuskaya, Sq. 4, Moscow A-47, U.S.S.R.

94. W. M. Stacey, Jr., School of Nuclear Engineering, Georgia Institute of Technology, Atlanta, GA 30332

95. L. D. Stewart, Princeton Plasma Physics Laboratory, P.0. Box 451, Princeton, NJ 08540

96. J. B. Taylor, Culham Laboratory, U.K. Atomic Energy, Authority, Abingdon, Oxon, OX14 3DB, United Kingdom

97. Thermonuclear Library, Japan Atomic Energy Research Institute, Tokai, Naka, Ibaraki, Japan

98. Francisco Verdaguer, Director, Division of Fusion, Junta de Energia Nuclear, Madrid 3, Spain

99. K. M. Zwilsky, Office of Fusion Energy, G-234, Department of Energy, Washington, DC 20545

100. Director, Research and Technical Support Division, Department of Energy, Oak Ridge Operations, P.O. Box E, Oak Ridge, TN 37830

101-335. Given distribution as shown in TID-4500, Magnetic Fusion Energy (Distribution Category UC-20a, d, and g: Plasma Systems, Fusion Systems, and 'lheoretical Plasma Physics) 